\title{
High-resolution time of flight neutron diffraction and magnetization studies of spin reorientation and polar transitions in $\mathrm{SmCrO3}$
}

\author{
Tusita Sau, Poonam Yadav, Shivani Sharma, Rajamani \\ Raghunathan, Pascal Manuel, Vaclav Petricek, U. P. Deshpande, \\ and N. P. Lalla
}

\section{Published version information}

Citation: T Sau et al. High-resolution time of flight neutron diffraction and magnetization studies of spin reorientation and polar transitions in $\mathrm{SmCrO3}$. Phys Rev B 103, no. 14 (2021): 144418

DOI: $10.1103 /$ PhysRevB.103.144418

This version is made available in accordance with publisher policies. Please cite only the published version using the reference above. This is the citation assigned by the publisher at the time of issuing the APV. Please check the publisher's website for any updates. 


\title{
High-resolution time of flight neutron diffraction and magnetization studies of spin reorientation and polar transitions in $\mathrm{SmCrO}_{3}$
}

\author{
Tusita Sau $\odot,{ }^{1}$ Poonam Yadav $\odot,{ }^{1}$ Shivani Sharma, ${ }^{2}$ Rajamani Raghunathan $\odot,{ }^{1}$ Pascal Manuel $\odot,{ }^{3}$ Vaclav Petricek ${ }^{4}$ \\ U. P. Deshpande $\odot{ }^{1}$ and N. P. Lalla ${ }^{1, *}$ \\ ${ }^{1}$ UGC-DAE Consortium for Scientific Research, Indore-452001, India \\ ${ }^{2}$ National High Magnetic Field Laboratory, Florida State University, Tallahassee, Florida 32310, USA \\ ${ }^{3}$ ISIS Facility, Rutherford Appleton Laboratory, Chilton, Didcot OX11 0QX, United Kingdom \\ ${ }^{4}$ Institute of Physics CAS, Na Slovance 1999/2, Praha, Czech Republic
}

(Received 4 November 2020; revised 2 February 2021; accepted 16 March 2021; published 12 April 2021)

\begin{abstract}
Rare-earth chromates have always been of interest due to temperature-induced magnetization reversal and spin-reorientation phase transitions (SRPTs). In orthochromates containing magnetic rare earths, the spin configuration is supposed to undergo a characteristic changeover across the SRPT followed by an independent ordering of rare-earth moments leading to polar order. However, due to the presence of nearly $14 \%$ of highly neutron-absorbing isotope ${ }^{149} \mathrm{Sm}$ in natural $\mathrm{Sm}$ based compounds, correct magnetic structure determination of $\mathrm{SmCrO}_{3}$ through neutron diffraction measurements has been a challenge. In the present study we investigate the pre- and post-SRPT spin configurations in well characterized $\mathrm{SmCrO}_{3}$ through time of flight neutron diffraction measurements carried out in zero field at the high-resolution high-flux WISH beam line of ISIS, in the United Kingdom. Magnetization measurement shows a canted antiferromagnetic phase transition at $T_{N 1}=192 \mathrm{~K}$, giving rise to a weak ferromagnetism, which undergoes a SRPT at $37 \mathrm{~K}$. Rietveld analysis of the neutron powder diffraction data shows that below $T_{N 1}=192 \mathrm{~K}$ the $\mathrm{Cr}^{3+}$ and $\mathrm{Sm}^{3+}$ moments order in a $P b^{\prime} n^{\prime} m: \Gamma_{4}\left(G_{x}, A_{y}, F_{Z} ; F_{Z}^{R}\right)$ spin configuration with their tiny ferromagnetic components $F_{Z}$ and $F_{Z}^{R}$, giving rise to weak ferromagnetism. Below $37 \mathrm{~K}$ the $P b^{\prime} n^{\prime} m: \Gamma_{4}\left(G_{x}, A_{y}, F_{Z} ; F_{Z}^{R}\right)$ configuration transforms to $\mathrm{Pbn}^{\prime} \mathrm{m}^{\prime}: \Gamma_{2}\left(F_{x}, C_{y}, \boldsymbol{G}_{\boldsymbol{Z}} ; F_{x}^{R}, \boldsymbol{C}_{y}^{\boldsymbol{R}}\right)$ as a result of continuous rotation of $\mathrm{Cr}^{3+}$ moments, while approaching SRPT below $T_{N 1}$. At still lower temperatures the $P b n^{\prime} m^{\prime}: \Gamma_{2}\left(F_{x}, C_{y}, \boldsymbol{G}_{\boldsymbol{Z}} ; F_{x}^{R}, \boldsymbol{C}_{\boldsymbol{y}}^{\boldsymbol{R}}\right)$ phase transforms to polar phases, either the $P 2_{1}^{\prime} 2_{1}^{\prime} 2_{1}: \Gamma_{26}\left(C_{x}, \boldsymbol{G}_{\boldsymbol{y}}, F_{z} ; \boldsymbol{C}_{\boldsymbol{x}}^{\boldsymbol{R}}, A_{y}^{R}, F_{z}^{R}\right)$ or the $P n^{\prime} a 2_{1}^{\prime}: \Gamma_{27}\left(F_{x}, C_{y}, \boldsymbol{G}_{z} ; F_{x}^{R}, \boldsymbol{C}_{\boldsymbol{y}}^{\boldsymbol{R}}, G_{z}^{R}\right)$ phase, as a result of independent antiferromagnetic ordering of $\mathrm{Sm}^{3+}$ moments at $T_{N 2}<4 \mathrm{~K}$ through $\mathrm{Sm}^{3+}-\mathrm{Sm}^{3+}$ direct interaction. Our result of the transformation of $\mathrm{SmCrO}_{3}$ from $\Gamma_{4}$ to $\Gamma_{2}$ below SRPT is in contradiction with the $\Gamma_{1}\left(A_{x}, \boldsymbol{G}_{\boldsymbol{y}}, C_{Z} ; C_{z}^{R}\right)$ spin configuration as reported in Tripathi et al. [Phys. Rev. B 96, 174421 (2017)]. This issue has been independently settled through ground-state energy calculation using spin-dependent density functional theory confirming the $\Gamma_{2}$ spin configuration to be of lower energy as compared to that of the $\Gamma_{1}$. The role of magnetocrystalline anisotropy in the occurrence of SRPT has been discussed.
\end{abstract}

DOI: 10.1103/PhysRevB.103.144418

\section{INTRODUCTION}

Rare-earth and transition-metal based distorted perovskite magnetic oxides, typified by Pbnm structure, have been of continued interest for many decades. Related studies have immensely enriched the basic and applied solid-state physics. The rare-earth $(R e)$ orthomanganites $\left(R e \mathrm{MnO}_{3}\right)$ inherit antisymmetric superexchange, giving rise to magnetism-induced ferroelectricity [1,2]. The orthoferrites $(\operatorname{ReFeO})_{3}[3,4]$ and orthochromites $\left(\mathrm{Re} \mathrm{CrO}_{3}\right)$ [5-7] together with multiferroicity, possess the intriguing property of magnetization reversal resulting in negative magnetization, and spin-reorientation phase transition (SRPT) [8-18]. The magnetic properties arising out of unusual phase transitions in $\mathrm{ReCrO}_{3}$ lead to their possible novel applications in fast magnetic switching devices [19-21] and magnetic refrigeration [22].

*Corresponding author: nplallaiuc82@gmail.com
The orthochromate $\mathrm{SmCrO}_{3}$ has recently emerged as a possible magnetoelectric multiferroic [23,24]. The anisotropic magnetic susceptibility and magnetic structure of $\mathrm{SmCrO}_{3}$ have been intensively studied by Gorodetsky et al. [25] and it has been proposed that below SRPT Cr moments undergo $\Gamma_{4}\left(G_{x}\right)$ to $\Gamma_{2}\left(G_{z}\right)$ spin-structure transition. Through sound velocity and specific-heat measurements on single crystals, they concluded the SRPT in $\mathrm{SmCrO}_{3}$ to be a second-order phase transition [25], which arises due to softening of the acoustic branch of the associated spin wave. However, the attempt of independent determination of the spin configurations in $\mathrm{SmCrO}_{3}$ across canted antiferromagnetic (CAFM) and SRPT, through a magnetic structural tool such as neutron scattering, remained missing until recently [26].

The orthochromites and orthoferrites both undergo two magnetic phase transitions; the one at relatively higher temperatures is a CAFM phase, and the other results out of SRPT at relatively lower temperatures. In most of the studies (White [8], Bertaut et al. [27,28], Bertaut [29] and Shamir et al. [30]) 
these two transitions, CAFM and SRPT, are attributed, respectively, to $\Gamma_{4}\left(G_{x}, A_{y}, F_{Z} ; F_{Z}^{R}\right)$ and $\Gamma_{2}\left(F_{x}, C_{y}, G_{Z} ; F_{x}^{R}, C_{y}^{R}\right)$ spin configurations, except a few, e.g., $\mathrm{DyFeO}_{3}[31]$, where $\Gamma_{4}$ transforms to $\Gamma_{1}$ across the SRPT. As indicated by Johnson et al. [32] and realized by Lee et al. [33,34] and Kuo et al. [35], to unravel the role of magnetic structure behind the novel physical properties, e.g., the magnetoelectric-multiferroic and magnetization reversal, knowledge of correct spin structure is vital.

Due to the presence of nearly $14 \%$ of highly neutronabsorbing isotope ${ }^{149} \mathrm{Sm}$ in natural $\mathrm{Sm}$ compounds, $\mathrm{SmCrO}_{3}$ has been challenging for its spin-structure determination using neutron scattering. Tripathi et al. [26] have tried to divulge the spin structure of $\mathrm{SmCrO}_{3}$ below SRPT through high-energy neutron powder diffraction (NPD) and have attributed it to a Pbnm $\Gamma_{1}\left(A_{x}, G_{y}, C_{Z} ; C_{z}^{R}\right)$ spin configuration. However, the majority of the spin configuration for SRPT is known to be $\Gamma_{2}\left(F_{x}, C_{y}, G_{Z} ; F_{x}^{R}, C_{y}^{R}\right)$ [8]. While using short-wavelength neutrons minimizes the absorption issues, a clear drawback of this approach is the low resolution of the scattering data, with mingled nuclear and magnetic peaks. This is quite likely to lead to a wrong conclusion (see Supplemental Material [36]). Looking into the importance of the knowledge of correct magnetic structure, we revisited the magnetic structure determination of $\mathrm{SmCrO}_{3}$ using the high-resolution high-flux time of flight (TOF) neutron scattering beam line WISH, at ISIS in the United Kingdom [37]. Our TOF NPD and magnetization studies reveal that the CAFM and SRPT phases of $\mathrm{SmCrO}_{3}$ possess the $\Gamma_{4}\left(\boldsymbol{G}_{\boldsymbol{x}}, A_{y}, F_{Z} ; F_{Z}^{R}\right)$ and $\Gamma_{2}\left(F_{x}, C_{y}, \boldsymbol{G}_{\boldsymbol{Z}} ; F_{x}^{R}, \boldsymbol{C}_{\boldsymbol{y}}^{\boldsymbol{R}}\right)$ spin configurations, respectively. The $\mathrm{Sm}^{3+}$ ions undergo a $G_{Z}^{R}$ type second-order AFM ordering at $T_{N 2}<4 \mathrm{~K}$ through $\mathrm{Sm}^{3+}-\mathrm{Sm}^{3+}$ direct interaction leading to a $\Gamma_{2}$ to $\Gamma_{27}$ phase transition of $\mathrm{SmCrO}_{3}$ with $P n^{\prime} a 2_{1}^{\prime}$ polar order.

\section{EXPERIMENT}

A polycrystalline sample of $\mathrm{SmCrO}_{3}$ was synthesized by a standard solid-state route. Stoichiometric amounts of dried $\mathrm{Sm}_{2} \mathrm{O}_{3}$ (99.99\%) and $\mathrm{Cr}_{2} \mathrm{O}_{3}(99.99 \%)$ were accurately weighed and ground for $\sim 8 \mathrm{~h}$ in an agate mortar and pestle. The mixture of reactants was calcined in air in a $\mathrm{MoSi}_{2}$ muffle furnace. The sample was reground after each firing sequence successively at $1000^{\circ} \mathrm{C}, 1100^{\circ} \mathrm{C}, 1200^{\circ} \mathrm{C}$, and $1300^{\circ} \mathrm{C}$ for $24 \mathrm{~h}$. Phase formation was monitored after each heating cycle using powder $x$-ray diffraction (XRD). Finally, the sample was pelletized in a disk shape under $80 \mathrm{kN} / \mathrm{m}^{2}$ pressure and sintered at $1350^{\circ} \mathrm{C}$ for $20 \mathrm{~h}$. The single-phase nature of the final sintered pellets was confirmed through roomtemperature (RT) XRD using a two-circle goniometer set in Bragg-Brentano geometry and mounted on a $\mathrm{Cu} K \alpha$ rotatinganode $\mathrm{x}$-ray generator working at $10 \mathrm{~kW}$. To measure the charge states of Sm and $\mathrm{Cr}$ in the compound, x-ray photoelectron spectroscopy (XPS) measurement was carried out using Al $K \alpha$ x rays with an ESCA system from SPECS GmbH. Energy-dispersive $\mathrm{x}$-ray analysis (EDS) analysis under scanning electron microscopy (SEM) was performed to determine the elemental composition of Sm and Cr. Dc magnetization vs temperature $(M-T)$ measurement, under a 500 Oe field, was done from $320 \mathrm{~K}$ down to $2 \mathrm{~K}$ under zero-field cool (ZFC), field cool cooling (FCC), and field cool warming (FCW) protocols using a SQUID magnetometer (Quantum Design Inc., USA). Isothermal $M-H$ measurements were also carried out at some selected temperatures up to $6 \mathrm{~T}$. To investigate the nuclear and magnetic structures of the compound, and its variation across the magnetic phase transitions, temperaturedependent TOF NPD was carried out at the time of flight beam line WISH at ISIS, UK. The high-resolution and high-flux design of WISH is quite suitable for studying such highabsorbing materials. For TOF NPD the powder sample was packed in a $3 \mathrm{~mm}$ vanadium capillary to minimize the absorption effects. The capability of the WISH beam line for such a high-absorbing sample was assured through a RT NPD measurement using just a RT candle holder. The low-temperature NPD data were collected during heating at 1.5, 25, 55, 90, $150,190,210$, and $250 \mathrm{~K}$ using a liquid-helium cryostat. The structural refinement of the RT XRD data, the symmetry analysis giving rise to the exhaustive list of possible irreducible representations (irreps) for magnetic moment configurations, and the nuclear and magnetic structure refinement were done using JANA-2006 [38].

\section{RESULT DISCUSSIONS}

\section{A. Phase purity characterization and magnetization studies}

Structural phase analysis of the as-prepared $\mathrm{SmCrO}_{3}$ sample was confirmed using XRD and TOF NPD both. Figure 1(a) shows the RT XRD data of the as-prepared $\mathrm{SmCrO}_{3}$ sample, Rietveld refined using the Pbnm space group. A perfect fit, with goodness of fit $(\mathrm{GOF})$ parameter $\sim 1.2$ and the presence of only $\sim 2 \mathrm{wt} \%$ impurity of $\mathrm{Cr}_{2} \mathrm{O}_{3}$ confirmed the formation of a nearly single-phase sample with Pbnm structure. Figure 1(b) shows the Rietveld refined RT TOF NPD data of the same $\mathrm{SmCrO}_{3}$ sample. The $\mathrm{Sm}, \mathrm{Cr}$, and the two oxygen atoms $\mathrm{O} 1$ and $\mathrm{O} 2$, were confirmed to occupy the $4 c, 4 b, 4 c$, and $8 d$ Wyckoff positions of the Pbnm structure, respectively. The refined RT crystal-structure parameters are given in Table I. The lengths of the $\mathrm{Cr}-\mathrm{O} 1(1.981 \AA)$ and $\mathrm{Cr}-\mathrm{O} 2(1.963 \AA)$ bonds and $\mathrm{Cr}-\mathrm{O} 1-\mathrm{Cr}\left(149.4^{\circ}\right)$ and $\mathrm{Cr}-\mathrm{O} 2-\mathrm{Cr}\left(149.7^{\circ}\right)$ bond angles, as determined from the fitted crystallographic parameters, were found to closely match with that of Xiang et al. $[39,40]$. However the $\mathrm{Cr}-\mathrm{O} 2-\mathrm{Cr}$ bond angle of $166^{\circ}$, the only structural parameter reported by Tripathi et al. [41], is found to be too off as compared to that of ours and Xiang et al. [39,40]; also see [36] for details. The perfect match between the RT crystal structure parameters, as obtained from the refinement of the XRD and NPD data, confirm the suitability of the WISH (ISIS, UK) beam line for the magnetic and structural phase-transition studies of $\mathrm{SmCrO}_{3}$ containing highly neutron-absorbing natural isotope ${ }^{149} \mathrm{Sm}$. The refined parameters are depicted as crystal structure models in the corresponding insets of Figs. 1(a) and 1(b). Figure 2 shows XPS spectra of spin-orbit doublets of (a) $\mathrm{Cr} 2 p_{1 / 2}$ and $2 p_{3 / 2}$ with binding energies 586.37 and $576.57 \mathrm{eV}$ and (b) $\mathrm{Sm} 3 d_{3 / 2}$ and $3 d_{5 / 2}$ with binding energies 1110.09 and $1083.12 \mathrm{eV}$, respectively. The measured binding energies for $\mathrm{Cr} 2 p$ and $\mathrm{Sm} 3 d$ match very well with the $\mathrm{Cr}^{3+}$ and $\mathrm{Sm}^{3+}$ charge states [42-45]. EDS analysis revealed 47 at. \% of Sm and 53 at. \% of $\mathrm{Cr}$, which, within the typical error of the EDS technique, is close to the intended 50\%-50\% composition of $\mathrm{Sm}$ and $\mathrm{Cr}$; see Ref. [36] for details of XPS and EDS analysis. 

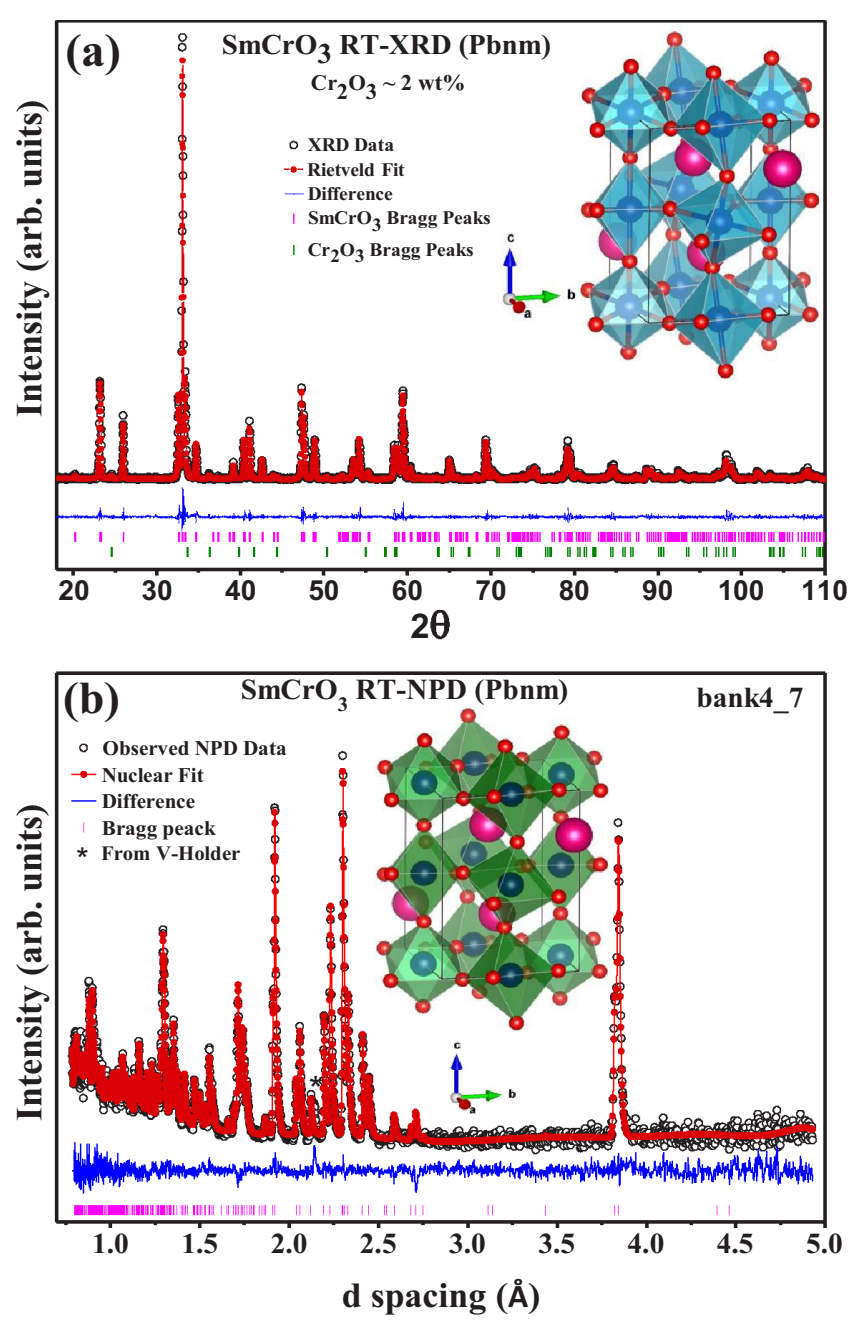

FIG. 1. Rietveld refined room-temperature (a) X-ray powder diffraction and (b) time of flight (TOF) neutron powder diffraction (NPD) data of the same $\mathrm{SmCrO}_{3}$ sample. The two-phase XRD refinement shows $\sim 2$ wt $\%$ of $\mathrm{Cr}_{2} \mathrm{O}_{3}$. XRD and NPD data have been refined using the Pbnm space group. The refined crystallographic parameters are given in Table I and the unit cell model of the crystal structure is shown in the corresponding insets. Highly symmetric $\mathrm{CrO}_{6}$ octahedra can be clearly seen.

Figure 3(a) shows the temperature dependence of dc magnetization $(M-T)$ of the studied $\mathrm{SmCrO}_{3}$. The features of $\mathrm{ZFC}$, FCC, and FCW $M-T$ curves are similar to that of Ref. [26]. The sharp jump in magnetization at $T_{N 1}=192 \mathrm{~K}$, resulting in a maximum of $\sim 0.07 \mu_{\mathrm{B}} /$ u.c. (unit cell) at low temperatures, signifies a weak-ferromagnetic (WF) transition. In the literature [46,47] related to rare-earth chromites such WF transitions are invariably attributed to the occurrence of canted antiferromagnetic (CAFM) order of $\mathrm{Cr}^{3+}$ moments, arising due to Dzyaloshinskii-Moriya (DM) interaction [48,49]. At $192 \mathrm{~K}$ the $\mathrm{Sm}^{3+}$ moments, however, still remain paramagnetic, but with decreasing temperature slowly get polarized under the exchange field of $\mathrm{Cr}^{3+}$ moments. This polarization is AFM in nature, and known [8,50,51] to cause continuous rotation of the $\mathrm{Cr}^{3+}$ moments leading to SRPT at $37 \mathrm{~K}$ in Fig. 3. The Curie-Weiss (CW) fit of the paramagnetic region of $\chi_{\mathrm{dc}}-T$ above $250 \mathrm{~K}$, as shown in the inset (i), gives an
TABLE I. Crystal structure parameters of $\mathrm{SmCrO}_{3}$ across the CAFM and SRPT as obtained from the Rietveld refinement of $300 \mathrm{~K}$ and low-temperature diffraction (XRD and TOF NPD) data.

\begin{tabular}{|c|c|c|c|c|}
\hline $\begin{array}{l}\text { Temperature } \\
\text { Data type } \\
\text { Space group }\end{array}$ & $\begin{array}{l}300 \mathrm{~K} \\
\text { XRD } \\
\text { Pbnm }\end{array}$ & $\begin{array}{c}300 \mathrm{~K} \\
\text { TOF NPD } \\
\text { Pbnm }\end{array}$ & $\begin{array}{c}90 \mathrm{~K} \\
\text { TOF NPD } \\
\text { Pbnm }\end{array}$ & $\begin{array}{c}1.5 \mathrm{~K} \\
\text { TOF NPD } \\
\text { Pbnm }\end{array}$ \\
\hline \multicolumn{5}{|c|}{ Lattice parameter } \\
\hline $\mathbf{a}(\AA)$ & $5.3655(7)$ & $5.3690(1)$ & $5.3634(8)$ & $5.3624(1)$ \\
\hline $\mathbf{b}(\AA)$ & $5.4988(1)$ & $5.5003(5)$ & $5.4911(7)$ & $5.4907(1)$ \\
\hline c $(\AA)$ & $7.6420(8)$ & $7.6460(7)$ & $7.6337(5)$ & $7.6316(3)$ \\
\hline \multicolumn{5}{|c|}{ Fractional atomic positions } \\
\hline $\operatorname{Sm}(4 c): x$ & 0.01001 & 0.01396 & 0.00944 & 0.01362 \\
\hline$: y$ & 0.05099 & 0.03780 & 0.03310 & 0.03649 \\
\hline$: z$ & 0.25 & 0.25 & 0.25 & 0.25 \\
\hline Occup: & 0.5 & 0.5 & 0.5 & 0.5 \\
\hline $\operatorname{Cr}(4 b): x$ & 0.5 & 0.5 & 0.5 & 0.5 \\
\hline$: y$ & 0 & 0 & 0 & 0 \\
\hline$: z$ & 0 & 0 & 0 & 0 \\
\hline Occup: & 0.5 & 0.5 & 0.5 & 0.5 \\
\hline $\mathbf{O}_{1}(4 c): x$ & -0.09641 & -0.08739 & -0.08472 & -0.08495 \\
\hline$: y$ & 0.48644 & 0.47765 & 0.47673 & 0.47692 \\
\hline$: z$ & 0.25 & 0.25 & 0.25 & 0.25 \\
\hline Occup: & 0.5 & 0.5 & 0.5 & 0.5 \\
\hline $\mathbf{O}_{2}(8 d): x$ & 0.29136 & 0.29675 & 0.29761 & 0.29733 \\
\hline$: y$ & 0.29636 & 0.29430 & 0.29495 & 0.29490 \\
\hline$: z$ & 0.05169 & 0.04519 & 0.04475 & 0.04443 \\
\hline Occup: & 1 & 1 & 1 & 1 \\
\hline \multicolumn{5}{|c|}{ Reliability factors } \\
\hline GOF & 2.01 & 0.97 & 1.69 & 1.68 \\
\hline$R_{P}$ & 9.50 & 2.12 & 2.32 & 2.34 \\
\hline$R_{w p}$ & 12.47 & 2.49 & 2.89 & 2.92 \\
\hline
\end{tabular}

effective moment of $7.8 \mu_{b}$, which is close to earlier reports [5,52]. The ZFC $M-T$ shows shallow minima below SRPT; see inset (ii). The FCW curve remains exactly reversible to FCC until $22 \mathrm{~K}$, but above it, follows an irreversible path showing a broad hysteresis, which finally merges at $T_{N 1}$. Irreversible FCC and FCW $M-T$ are common to most orthochromates comprising magnetic rare earths [10-12,14,16]. However, the details of irreversibility keep changing. For example, in $\mathrm{SmCrO}_{3}$, as shown in the above, the FCW magnetization remains lower than that of the $\mathrm{FCC}$, but for $\mathrm{GdCrO}_{3}$ [11] and $\mathrm{TmCrO}_{3}$ [12] it is opposite; the FCW magnetization becomes higher than that of the FCC. Figures 3(b) and 3(c) show $M-H$ variation at 25 and $60 \mathrm{~K}$ confirming the presence of weakferromagnetic domains with finite remanence and coercivity.

\section{B. Low-temperature neutron diffraction studies}

Figure 4(a) shows the temperature-dependent TOF NPD patterns of the $\mathrm{SmCrO}_{3}$ sample collected during warming at selected temperatures from 1.5 to $250 \mathrm{~K}$. Collection of each pattern was started after stabilizing the temperature within 0.1 $\mathrm{K}$. As discussed above, the peaks of the patterns were successfully indexed with $\mathrm{Pbnm}$ structure having distorted perovskite 

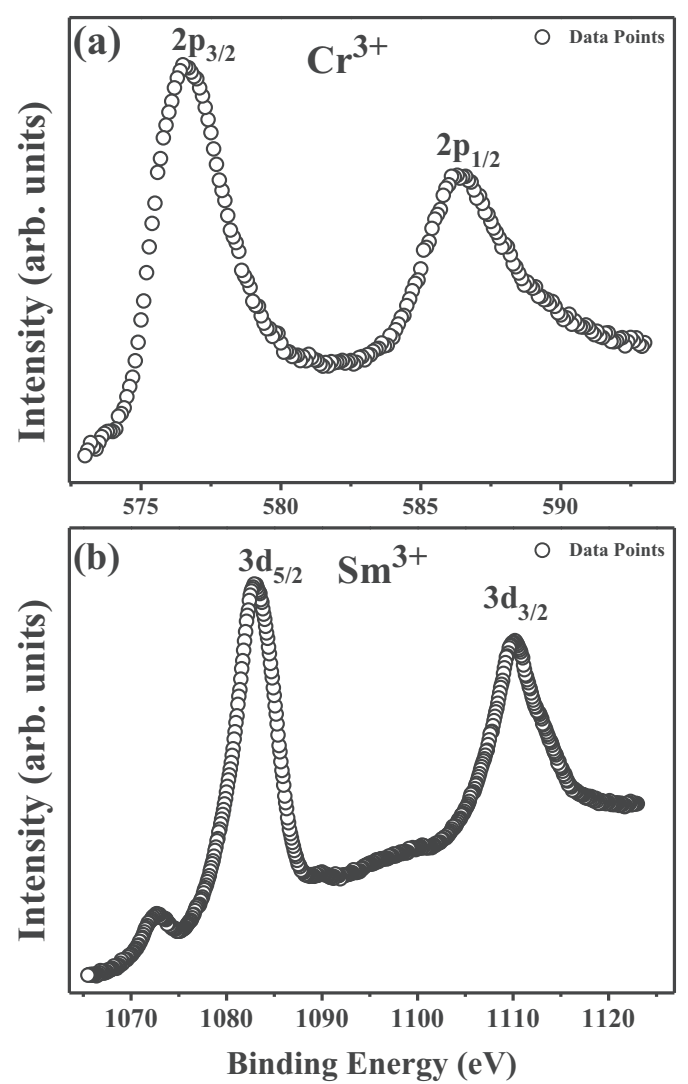

FIG. 2. X-ray photoelectron spectroscopy peaks of spin-orbit split doublets of (a) $\mathrm{Cr} 2 p$ and (b) $\mathrm{Sm} 3 d$. The measured binding energy (BE) positions of the peak maxima of (a) $\mathrm{Cr} 2 p_{1 / 2}$ and $2 p_{3 / 2}$ are 586.37 and $576.57 \mathrm{eV}$ and that for (b) $\mathrm{Sm} 3 d_{3 / 2}$ and $3 d_{5 / 2}$ are 1110.09 and $1083.12 \mathrm{eV}$, respectively. These BE values of doublets match very well with the reported (a) $\mathrm{Cr}^{3+}$ and (b) $\mathrm{Sm}^{3+}$ charge states.

orthorhombic lattice with parameters defined as $a \mathrm{o}=\sqrt{ } \mathbf{2} a_{p}$, $b_{o}=\sqrt{ } \mathbf{2} a_{p}$, and $c_{o}=\mathbf{2} a_{p}$, where $a_{p}$ is the pseudo-perovskite lattice parameter. The indices of the major diffraction peaks are indicated. Figures 4(b) and 4(c) are the enlarged views of the $(002) /(110)$ and $(101) /(011)$ peaks. It can be clearly noticed that, while the (002) and (110) set of peaks remains nearly unchanged at all temperatures from 1.5 to $250 \mathrm{~K}$, the (101) and (011) set of peaks appears only below $T_{N}$. As shown in the inset of Fig. 4(c), the (101) and (011) set of peaks undergoes changes in height in a nonmonotonous way. Close below the $T_{N}$, the peak heights first increase sharply, (011) remaining higher than (101), and then get slowly saturated until $55 \mathrm{~K}$. At temperatures below $55 \mathrm{~K}$ the nature of the peak height variation gets reversed, indicating commencement of some phase transition. The intensity of the (011) peak now drops sharply, while that of (101) increases sharply, and across SRPT, at $25 \mathrm{~K}$, both get nearly matched. At $1.5 \mathrm{~K}$ the intensity of (011) again becomes higher by $\sim 10 \%$ than that of the (101).

As confirmed through the refinement of the RT NPD pattern the (002) and (110) peaks in Fig. 1(b), and all other peaks below these, arise out of coherent nuclear scattering of neutrons from the crystal structure. The (101) and (011) peaks, appearing below $T_{N 1}$, indicate a phase transition below $T_{N 1}$.
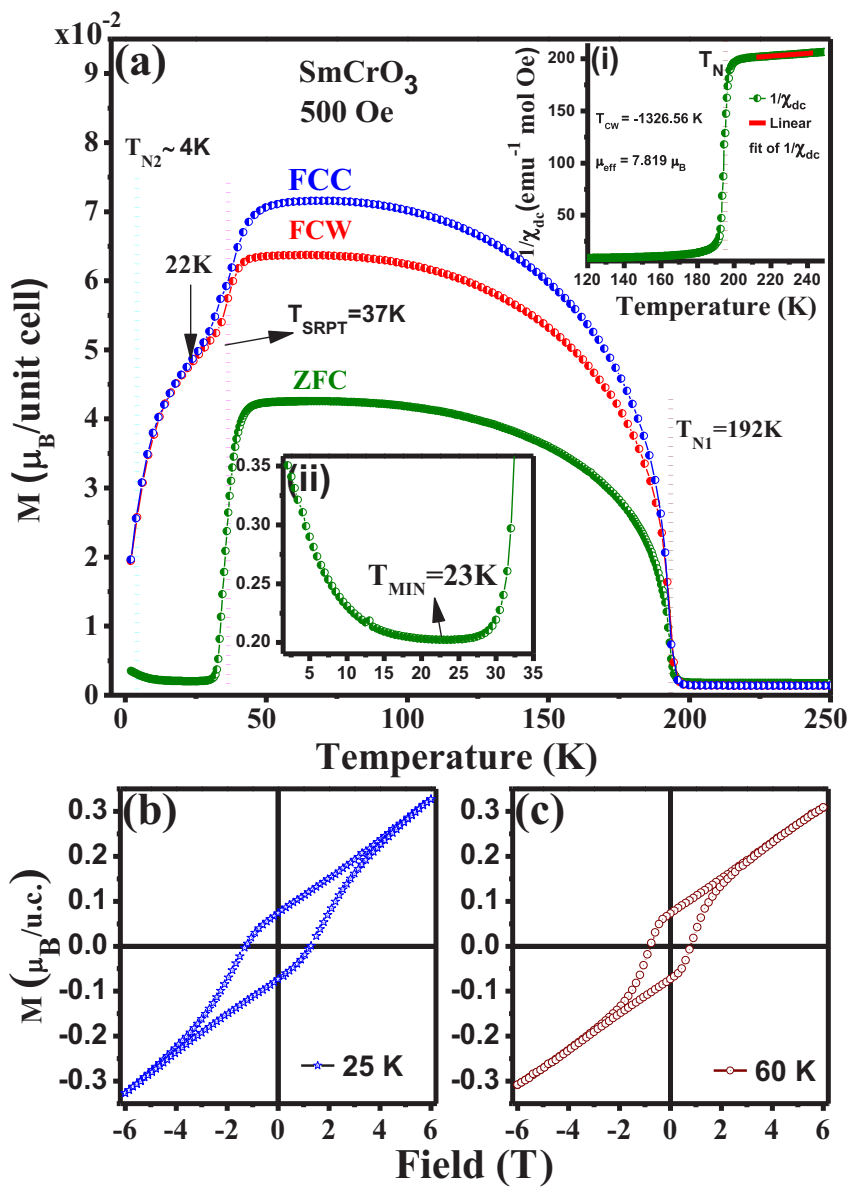

FIG. 3. (a) Zero-field cool (ZFC), field-cooled cooling (FCC), and field-cooled warming (FCW) dc-magnetization data of $\mathrm{SmCrO}_{3}$ measured under $500 \mathrm{Oe}$, showing occurrence of weak-ferromagnetic (WF) transition at $192 \mathrm{~K}$ due to canted antiferromagnetic (CAFM) ordering. The sharp drop in magnetization at 37 and $\sim 10.6 \mathrm{~K}$ are due to spin-reorientation phase transition (SRPT) and commencement of $\mathrm{Sm}$ AFM ordering due to direct $\mathrm{Sm}-\mathrm{Sm}$ interaction. Inset (i) shows $\mathrm{CW}$ fit and (ii) highlights the occurrence of magnetization minima at $\sim 23 \mathrm{~K}$. (b,c) show M-H variation at 25 and $60 \mathrm{~K}$, respectively.

Nuclear Rietveld refinement of the NPD data collected below $T_{N 1}$ confirmed that except for (101) and (011), all the diffraction peaks below these get fully accounted by the Pbnm crystal structure. The (101) and (011) peaks appear due to magnetic ordering giving rise to the CAFM phase at $T_{N 1}=192 \mathrm{~K}$. Since (101) and (011) are extinct for the Pbnm space group, their occurrence conveys that the magnetic ordering at $T_{N 1}$ lowers the net symmetry of the crystal structure, i.e., the symmetry of nuclear and spin structures both combined, below Pbnm.

Since the magnetic peaks are getting indexed as (101) and (011) based on the Pbnm parent unit cell basis vectors, the magnetic ordering vector is $\mathbf{k}=(0,0,0)$ (or just $\mathbf{k}=0)$ type. The occurrence of various possible spin configurations for $\mathbf{k}=0$ magnetic ordering in a Pbnm structure is well studied and has been very successfully applied to rareearth orthomanganites, ferrites, and chromates [27-30,5355]. There are eight possible spin configurations for $\mathbf{k}=0$ magnetic ordering of a Pbnm structure with magnetic ions at 


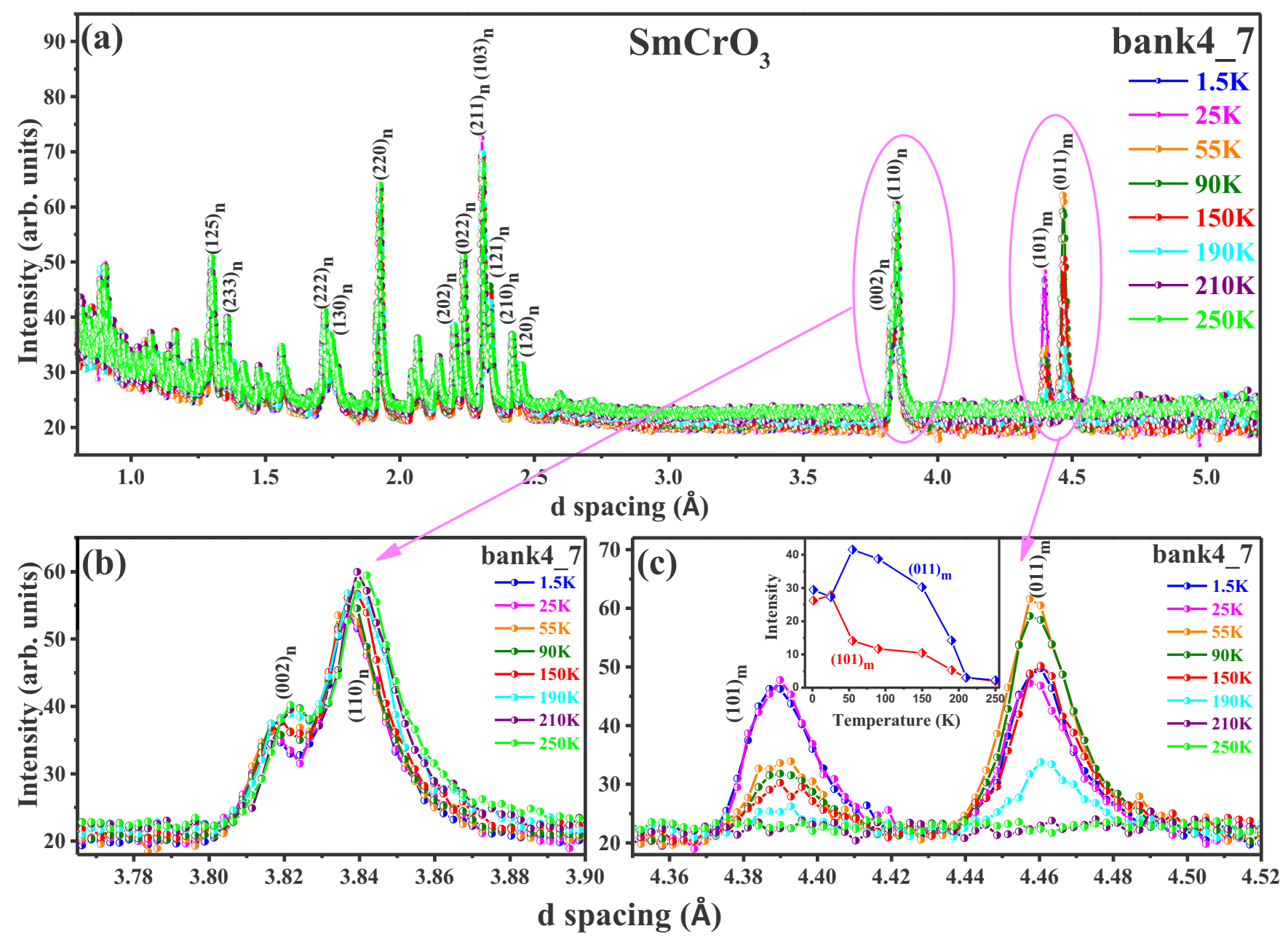

FIG. 4. (a) Stack of temperature-dependent TOF-NPD data taken during warming. (b,c) show, respectively, the enlarged views of the patterns corresponding to $(002)_{\mathrm{n}}$ and $(110)_{\mathrm{n}}$ nuclear scattering peaks and $(101)_{\mathrm{m}}$ and $(011)_{\mathrm{m}}$ magnetic scattering peaks. The temperature variation of the $(101)_{\mathrm{m}}$ and $(011)_{\mathrm{m}}$ magnetic scattering peaks indicates the change in spin configuration across paramagnetic to CAFM and CAFM to SRPT phase transitions.

$4 b$ and $4 c$ sites; see Table II $[29,30]$. As can be seen from Table II, out of the eight possible irreps, only four $-\Gamma_{1}, \Gamma_{2}$, $\Gamma_{3}$, and $\Gamma_{4}$-allow for the finite value to the ordered moment of a magnetic ion at the $4 b$ site. The other four $-\Gamma_{5}, \Gamma_{6}$, $\Gamma_{7}$, and $\Gamma_{8}$ (or $\Gamma_{1-}, \Gamma_{2-}, \Gamma_{3-}$, and $\left.\Gamma_{4-}\right)$-do not allow any ordered moment at the $4 b$ site.

TABLE II. The unique set of irreps and the corresponding magnetic basis vectors for the moment configurations of magnetic ions at ( $4 b)$ and $(4 c)$ sites in a Pbnm structure that has undergone $\mathbf{k}=(0,0,0)$ ordering.

\begin{tabular}{lccc}
\hline \hline Space group & irreps & $(4 b) \mathrm{Cr}$ & $(4 c) \mathrm{Sm}$ \\
\hline$P b n m$ & $\Gamma_{1}$ & $A_{x} G_{y} C_{z}$ & $--C_{z}$ \\
$P b n^{\prime} m^{\prime}$ & $\Gamma_{2}$ & $F_{x} C_{y} G_{z}$ & $F_{x} C_{y}-$ \\
$P b^{\prime} n m^{\prime}$ & $\Gamma_{3}$ & $C_{x} F_{y} A_{z}$ & $C_{z} F_{y}-$ \\
$P b^{\prime} n^{\prime} m$ & $\Gamma_{4}$ & $G_{x} A_{y} F_{z}$ & $--F_{z}$ \\
$P b^{\prime} n^{\prime} m^{\prime}$ & $\Gamma_{5}$ & --- & $G_{x} A_{y}-$ \\
$P b^{\prime} n m$ & $\Gamma_{6}$ & --- & $--A_{z}$ \\
$P b n^{\prime} m$ & $\Gamma_{7}$ & --- & $--G_{z}$ \\
$P b n m^{\prime}$ & $\Gamma_{8}$ & --- & $A_{x} G_{y}-$ \\
\hline \hline
\end{tabular}

The spin configurations of $\mathrm{Cr}^{3+}$ and $\mathrm{Sm}^{3+}$ magnetic ions were determined through combined nuclear and magnetic structure refinement using JANA-2006. JANA-2006 provides tools such as "REPRESENTATION ANALYSIS OF MAGNETIC STRUCTURE" and ISODISTORT [56]; both generate an exhaustive list of irreps for all the possible spin configurations for a given nuclear structure and magnetic ordering propagation vector. The ISODISTORT option can be used to generate magnetic structure crystal information files (CIFs) with desired superimposition of more than one irrep. In the present case the CAFM ordering takes place at $192 \mathrm{~K}$. Since it is quite unphysical to consider $\mathrm{Sm}^{3+}$ getting independently ordered at such a high temperature, the observed CAFM order is fully accounted to $\mathrm{Cr}^{3+}$ ordering arising from $\mathrm{Cr}^{3+}-$ $\mathrm{O}^{2-}-\mathrm{Cr}^{3+}$ superexchange. Therefore, for magnetic Rietveld refinement, out of the eight possible spin configurations, only those were selected, which allowed a finite moment at the $4 b$ site $\left(\mathrm{Cr}^{3+}\right)$, i.e., $P b n m: \Gamma_{1}\left(A_{x}, G_{y}, C_{Z} ; C_{z}^{R}\right), P b n^{\prime} m^{\prime}$ : $\Gamma_{2}\left(F_{x}, C_{y}, G_{Z} ; F_{x}^{R}, C_{y}^{R}\right), P b^{\prime} n m^{\prime}: \Gamma_{3}\left(C_{x}, F_{y}, A_{Z} ; C_{x}^{R}, F_{y}^{R}\right)$, and $P b^{\prime} n^{\prime} m: \Gamma_{4}\left(G_{x}, A_{y}, F_{Z} ; F_{Z}^{R}\right)$ [27-29]. All the components of the selected irrep were set free during the refinement iterations. Since these irreps are responsible only for the 
occurrence of magnetic peaks, to decide upon the correct spin structure, we monitored the fit quality of (101) and (011) magnetic peaks once the refinement iterations converged to a stable GOF parameter for the selected irrep. Keeping in view the highly neutron-absorbing nature of ${ }^{149} \mathrm{Sm}$, the pseudo absorption correction was refined for obtaining the best fit. For details on the pseudo absorption correction see the Supplemental Material [36]. At a given temperature the correction factor was found to be the lowest for the irrep giving the best fit and ranged from 0.36 to 0.42 .

Figures 5(a) and 5(b) compare the fit quality of the refinement of the magnetic peaks collected at 25 and $90 \mathrm{~K}$, respectively. Each of Figs. 5(a) and 5(b) has four panels, each depicting the quality of magnetic fittings corresponding to the irreps $\Gamma_{1}, \Gamma_{2}, \Gamma_{3}$, and $\Gamma_{4}$, as indicated in the panels. It can be seen that the best fit for the 25 and $90 \mathrm{~K}$ TOF NPD data of $\mathrm{SmCrO}_{3}$ correspond to $\Gamma_{2}\left(F_{x}, C_{y}, \boldsymbol{G}_{z} ; F_{x}^{R}, \boldsymbol{C}_{\boldsymbol{y}}^{\boldsymbol{R}}\right)$ and $\Gamma_{4}\left(\boldsymbol{G}_{\boldsymbol{x}}, A_{y}, F_{z} ; F_{z}^{R}\right)$, respectively. A review of the standard uncertainties (s.u.) corresponding to the values of moments of each component of $\Gamma_{2}$ and $\Gamma_{4}$ irreps, while the components were set free to iterate, revealed that the ferromagnetic components $F_{x}$ of $\mathrm{Cr}$ and $F_{z}^{R}$ of Sm were very small, while the corresponding s.u. were many times larger; see Table SM2 [36]. The large s.u. basically indicated the insignificance of these components, and hence in the next approach the $F_{x}$ and $F_{z}^{R}$ were constrained to zero before iteration. Since these components were already very small the constrained refinement resulted in only a very slight improvement in the profile of the (101) and (011) magnetic peaks. The GOF also lowered only slightly; see Table SM3 [36]. Here it should be noted that the weak ferromagnetism, as experimentally observed through $M-T$ and $M-H$ in Figs. 3(a)-3(c), confirms the presence of $F_{x}$ and $F_{z}^{R}$ components. The results of the constrained refinement physically signify that the values of the $F_{x}$ and $F_{z}^{R}$ components, which are necessary to give rise to weak ferromagnetism in the CAFM phase, are too small to be significantly realized under the given statistics of the TOF NPD data. Thus, as indicated by the bold letters [55], the major component of CAFM ordering below $192 \mathrm{~K}$ corresponds to $\boldsymbol{G}_{\boldsymbol{x}}$ type and that of the SRPT to $\boldsymbol{G}_{\boldsymbol{z}}$ type. As can be inferred from the irrep in Table II the realization of just even the $\boldsymbol{G}_{\boldsymbol{x}}$ and $\boldsymbol{G}_{\boldsymbol{z}}$ components, for a moment siting at the $4 b$ site in a Pbnm phase, uniquely confirms the presence of $\Gamma_{4}$ and $\Gamma_{2}$ spin configurations, respectively. Here it appears imperative to discuss that to make the structure refinement comparison equal between the $\Gamma_{1}$ and $\Gamma_{2}$ irreps, the constrained refinement approach was followed also for the $\Gamma_{1}$ irrep selected for that of $25 \mathrm{~K}$ TOF NPD data. However, there was no consequent change in the comparison already depicted in Figs. 5(a) and 5(b).

As seen in Fig. 3(a), the reversible drop in FCC and FCW magnetization below $22 \mathrm{~K}$ is due to the emergence of $\mathrm{Sm}^{3+}-\mathrm{Sm}^{3+}$ interaction leading to an independent secondorder Sm AFM ordering at low temperatures. Specific-heat measurement does clearly show Sm ordering [25] below $4 \mathrm{~K}$. Occurrence of additional magnetic ordering at $1.5 \mathrm{~K}$ is expected to modify the intensity of the magnetic peaks. Figure 6(a) shows the comparison of peak intensities of the $(101)_{\mathrm{m}}$ and $(011)_{\mathrm{m}}$ magnetic peaks at $1.5 \mathrm{~K}$. It can be seen that the height of the $(011)_{\mathrm{m}}$ magnetic peak at $1.5 \mathrm{~K}$ is lower by $\sim 10 \%$ w.r.t. that at $25 \mathrm{~K}$. Yamaguchi and Tsushima [4] have shown that additional ordering of Sm makes the $P b n^{\prime} m^{\prime}$ : $\Gamma_{2}\left(F_{x}, C_{y}, \boldsymbol{G}_{z} ; F_{x}^{R}, \boldsymbol{C}_{\boldsymbol{y}}^{\boldsymbol{R}}\right)$ magnetic structure degenerate. Two possibilities result; the first arises from the superposition of $P b n^{\prime} m^{\prime}: \Gamma_{2}$ and $P b^{\prime} n m: \Gamma_{6}$, leading to $\Gamma_{26}$, and the second from the superposition of $P b n^{\prime} m^{\prime}: \Gamma_{2}$ and $P b n^{\prime} m: \Gamma_{7}$, leading to $\Gamma_{27}$. As an effect of these superimpositions the nonpolar space group Pbnm transforms to polar space groups $P 22_{1} 2_{1} 2_{1}$ and $P n a 2_{1}$. The $P b n^{\prime} m^{\prime}: \Gamma_{2}\left(F_{x}, C_{y}, \boldsymbol{G}_{z} ; F_{x}^{R}, \boldsymbol{C}_{\boldsymbol{y}}^{\boldsymbol{R}}\right)$ transforms, respectively, either to $P 2_{1}^{\prime} 2_{1}^{\prime} 2_{1}:\left(C_{x}, \boldsymbol{G}_{\boldsymbol{y}}, F_{z} ; \boldsymbol{C}_{\boldsymbol{x}}^{\boldsymbol{R}}, A_{y}^{R}, F_{z}^{R}\right)$ or $P n^{\prime} a 2_{1}^{\prime}:\left(C_{x}, F_{y}, \boldsymbol{G}_{z} ; \boldsymbol{C}_{\boldsymbol{x}}^{\boldsymbol{R}}, \boldsymbol{F}_{\boldsymbol{y}}^{\boldsymbol{R}}, G_{z}^{R}\right)$. Please note that permutation of the magnetic ordering types in the above representations follows that of the corresponding lattice parameters. Following the ISOSDISTORT option in JANA-2006 the magnetic CIFs corresponding to $\Gamma_{26}$ and $\Gamma_{27}$ were generated and used for refinement of the $1.5 \mathrm{~K}$ TOF NPD data. As described in the above, unconstrained and constrained magnetic refinements were carried out. Tables SM 3(a) and SM3(b) [36] present the components of the refined moments and the corresponding errors for unconstrained and constrained magnetic refinements. The unconstrained refinement results in identical GOF, 1.68. Figures 6(b)-6(d) show the comparison of unconstrained refinement of the major magnetic peaks at $1.5 \mathrm{~K}$; notice the encircled tip region of the right-side magnetic ordering peak. After close evaluation it is realized that $\Gamma_{27}$, i.e., $P n^{\prime} a 2_{1}^{\prime}:\left(C_{x}, F_{y}, \boldsymbol{G}_{z} ; \boldsymbol{C}_{\boldsymbol{x}}^{\boldsymbol{R}}, \boldsymbol{F}_{\boldsymbol{y}}^{\boldsymbol{R}}, G_{z}^{R}\right)$, provides the better fit. However, after setting the moments with large errors to zero, in both cases $\Gamma_{26}$ and $\Gamma_{27}$, the constrained refinement yields lower GOF of 1.64 for $\Gamma_{26}$ as compared to 1.66 for $\Gamma_{27}$. This implies that under the given statistics of the TOF NPD data differentiation between $\Gamma_{26}$ and $\Gamma_{27}$ phases can not be done.

The typical fit quality of the Rietveld refined full NPD patterns, corresponding to $1.5,25$, and $90 \mathrm{~K}$, are shown in Figs. 7(a) and 7(c), respectively. The refined magnetic structure models corresponding to $1.5 \mathrm{~K}\left(\Gamma_{26}\right.$ and $\left.\Gamma_{27}\right), 25 \mathrm{~K}$ $\left(\Gamma_{2}\right)$, and $90 \mathrm{~K}\left(\Gamma_{4}\right)$ are shown in Figs. $8(\mathrm{a})-8(\mathrm{~d})$. In the $\Gamma_{4}$ magnetic structure model shown in Fig. 8(d), corresponding to $90 \mathrm{~K}$, the ordered $\mathrm{Sm}$ moment has not been shown due to its insignificant value. The comparison of the fit quality of the (101) and (011) magnetic peaks of TOF NPD data collected at other temperatures is shown in the Supplemental Material [36]. All these results confirm that below $T_{N 1}=192 \mathrm{~K}$ $\mathrm{SmCrO}_{3}$ undergoes a paramagnetic to CAFM transition comprising $P b^{\prime} n^{\prime} m: \Gamma_{4}\left(\boldsymbol{G}_{\boldsymbol{x}}, A_{y}, F_{z} ; F_{z}^{R}\right)$ spin configuration. On lowering the temperature, at $\sim 37 \mathrm{~K}$ the $P b^{\prime} n^{\prime} m: \Gamma_{4}$ spin configuration transforms to $P b n^{\prime} m^{\prime}: \Gamma_{2}\left(F_{x}, C_{y}, \boldsymbol{G}_{z} ; F_{x}^{R}, \boldsymbol{C}_{\boldsymbol{y}}^{\boldsymbol{R}}\right)$. Below $37 \mathrm{~K}$ the $\mathrm{Cr}^{3+}$ and $\mathrm{Sm}^{3+}$ moments undergo spinreorientation transition such that the net FM component now orients along the a axis, $F_{x}$. The presence of such magnetic components and their changeover during SRPT have been realized in bulk magnetization measurements on single crystal [17,25,57]. At temperatures below SRPT the $P b n^{\prime} m^{\prime}$ : $\Gamma_{2}\left(F_{x}, C_{y}, \boldsymbol{G}_{z} ; F_{x}^{R}, \boldsymbol{C}_{\boldsymbol{y}}^{\boldsymbol{R}}\right)$ phase transforms to the polar phase, either $P 2_{1}^{\prime} 2_{1}^{\prime} 2_{1}: \Gamma_{26}\left(C_{x}, \boldsymbol{G}_{\boldsymbol{y}}, F_{z} ; \boldsymbol{C}_{\boldsymbol{x}}^{\boldsymbol{R}}, A_{y}^{R}, F_{z}^{R}\right)$ or $P n^{\prime} a 2_{1}^{\prime}: \Gamma_{27}$ $\left(F_{x}, C_{y}, \boldsymbol{G}_{z} ; F_{x}^{R}, \boldsymbol{C}_{\boldsymbol{y}}^{\boldsymbol{R}}, G_{z}^{R}\right)$, due to Sm-Sm independent AFM ordering. Figure 9 shows temperature dependence of the ordered moment values of $\mathrm{Cr}^{3+}$ and $\mathrm{Sm}^{3+}$ ions as obtained from the constrained refinement of the magnetic structure. The 

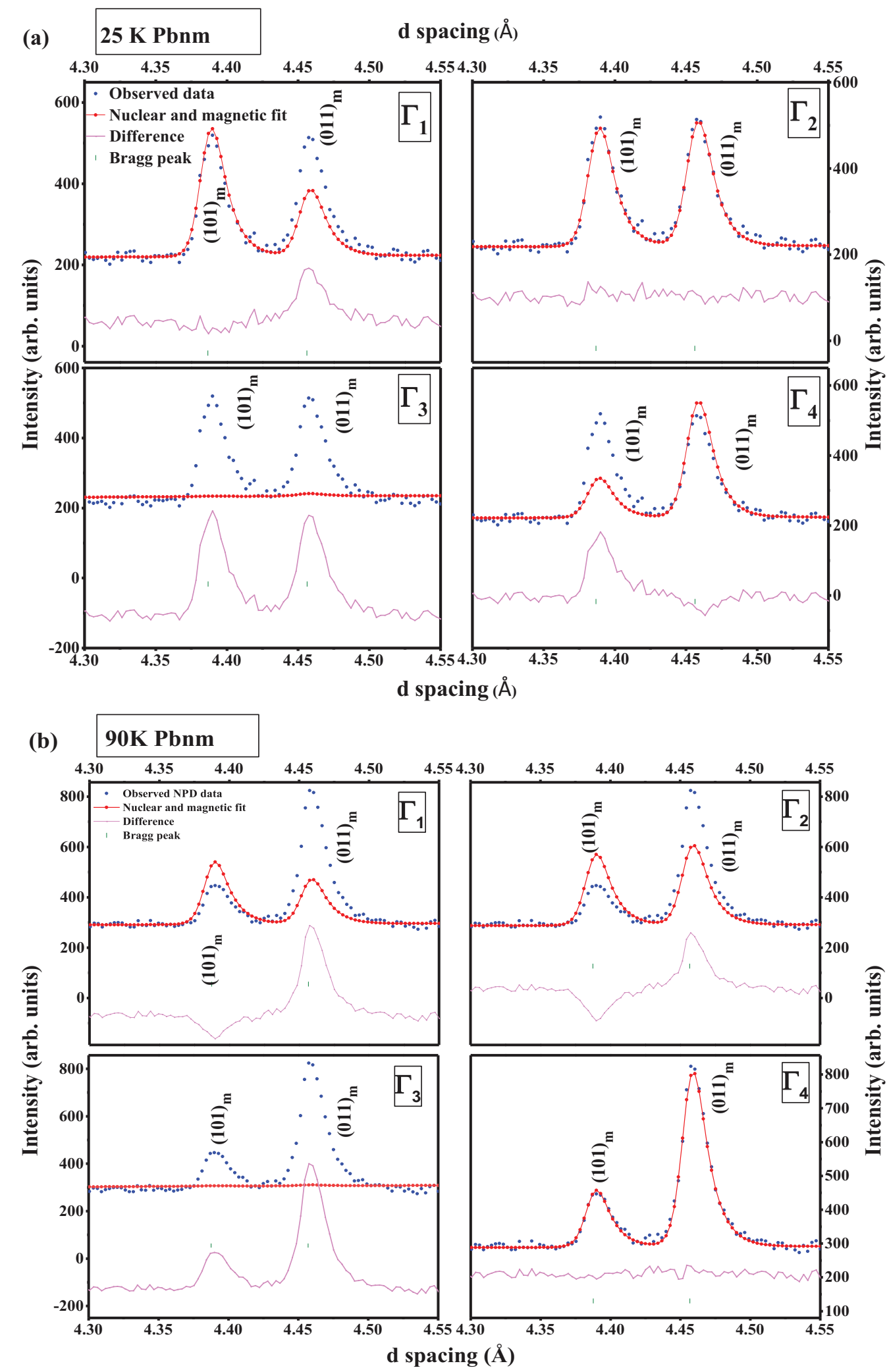

FIG. 5. Comparison of Rietveld fit quality of the refinement of $(101)_{\mathrm{m}}$ and $(011)_{\mathrm{m}}$ magnetic scattering peaks using spin configurations $\Gamma_{1}$, $\Gamma_{2}, \Gamma_{3}$, and $\Gamma_{4}$. The comparison shows that (a) after SRPT, at $1.5 \mathrm{~K}$ the spin structure belongs to $\Gamma_{2}$ and (b) at $90 \mathrm{~K}$, i.e., below the CAFM transition, the spin structure belongs to $\Gamma_{4}$. 


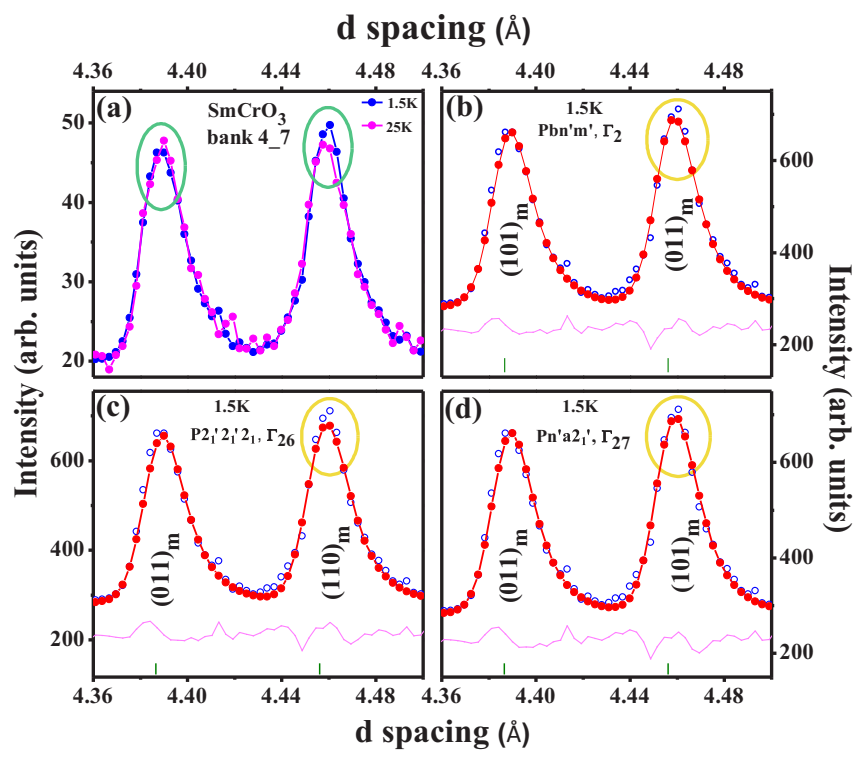

FIG. 6. Comparison of (a) reverse intensity change in the main magnetic scattering peaks at $1.5 \mathrm{~K}$ as compared to that at $25 \mathrm{~K}$, and their Rietveld fitting done using (b) $\Gamma_{2}$, (c) $\Gamma_{26}$, and (d) $\Gamma_{27}$ spin configurations. The comparison shows that the $\Gamma_{27}$ spin configuration matches best with the $1.5 \mathrm{~K}$ magnetic scattering peaks.

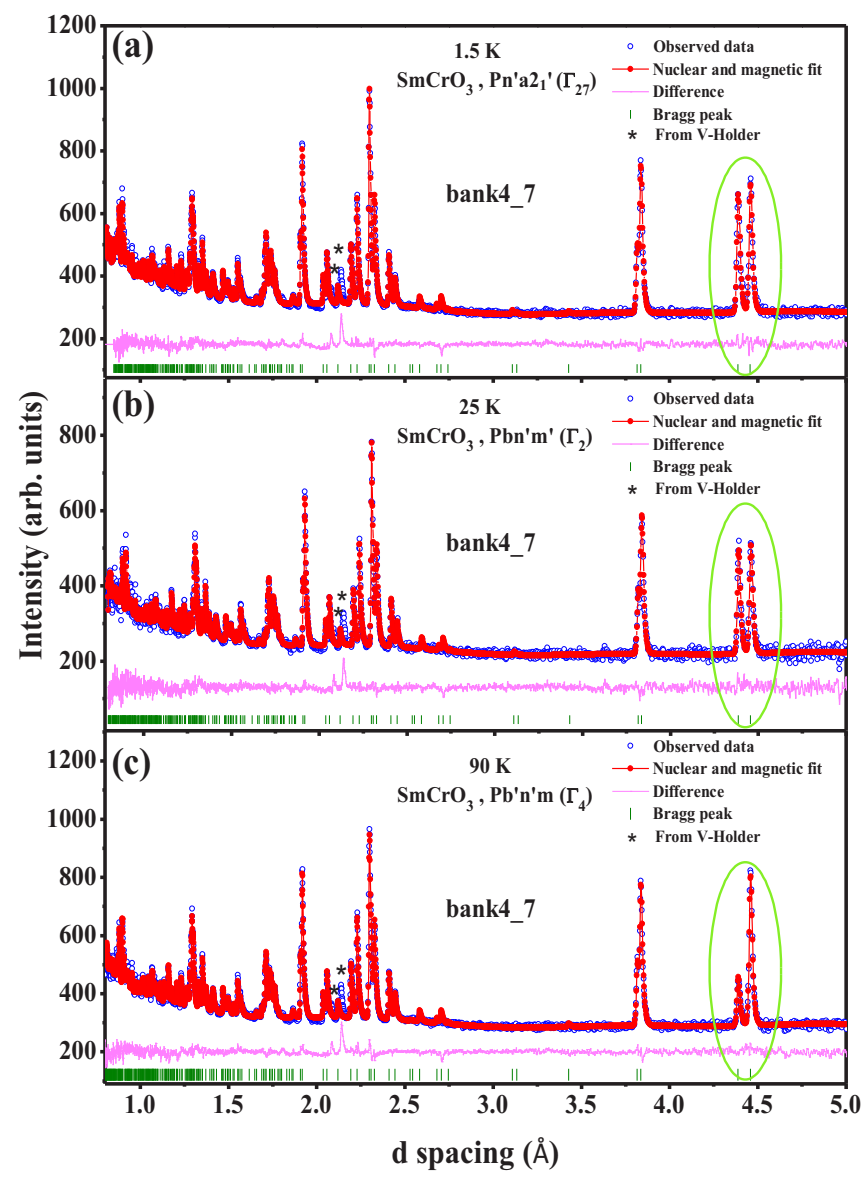

FIG. 7. Rietveld refined full TOF NPD patterns collected at (a) $1.5 \mathrm{~K}$, (b) $25 \mathrm{~K}$, and (c) $90 \mathrm{~K}$ showing the typical fit quality of the refinement based on $\Gamma_{27}, \Gamma_{2}$, and $\Gamma_{4}$ irreps, respectively.
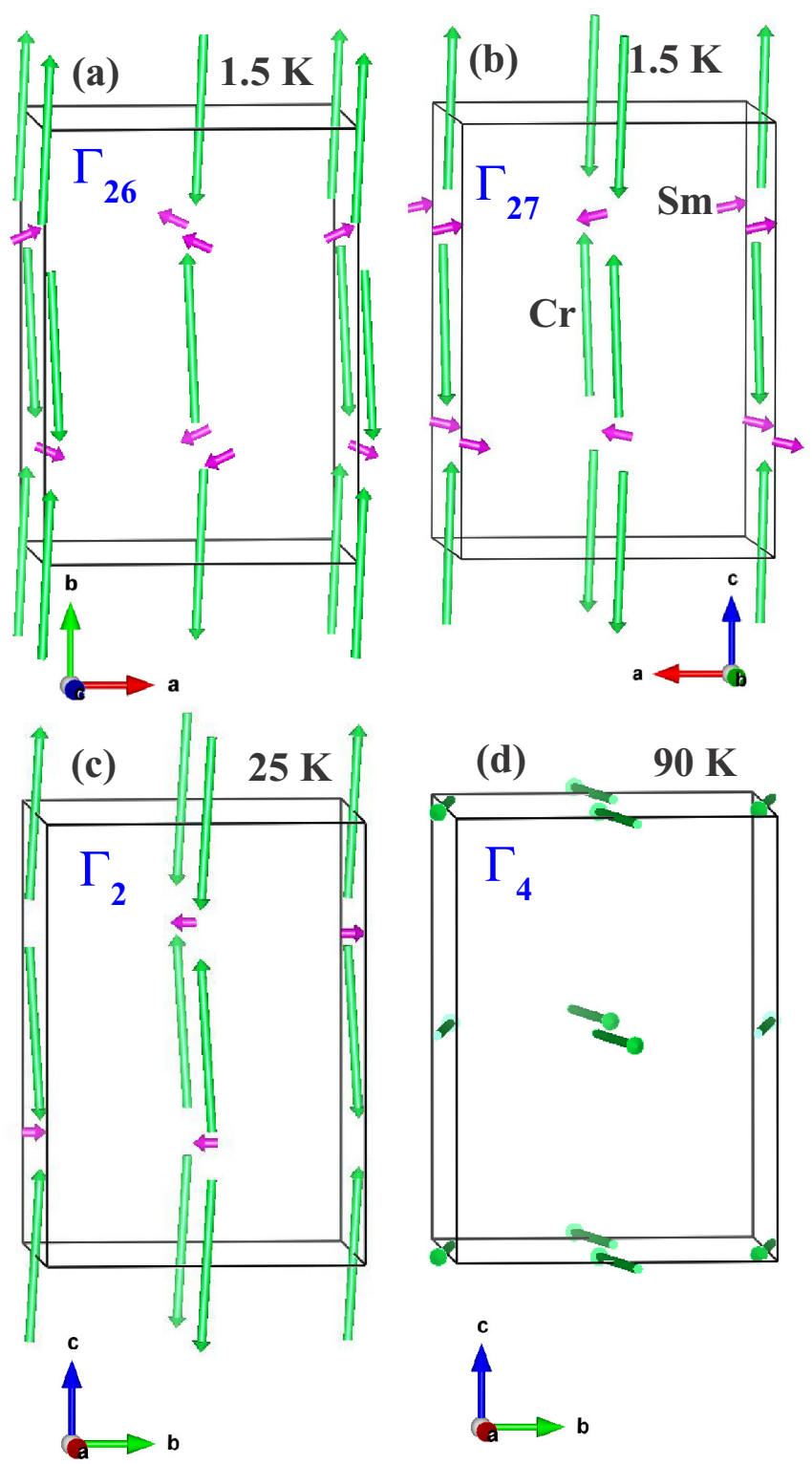

FIG. 8. (a) $\Gamma_{26}$ and (b) $\Gamma_{27}$ spin-structure models made based on the unconstrained refinement. It reveals spin configurations showing Sm-Sm direct AFM ordering over the $\Gamma_{2}$ ordering at $1.5 \mathrm{~K}$. (c) $\Gamma_{2}$ ordering at $25 \mathrm{~K}$, after SRPT at $37 \mathrm{~K}$ and (d) $\Gamma_{4}$ ordering at $90 \mathrm{~K}$, below the CAFM transition as obtained through constrained refinement.

observed moment of $\mathrm{Cr}^{3+}$ in its most ordered state at $1.5 \mathrm{~K}$ is $\sim 2.9 \mu_{b}$, which is very close to the expected spin-only value of $3 \mu_{b}$. The observed most ordered value of $\mathrm{Sm}^{3+}$ moment is $0.63 \mu_{b}$, which is slightly lower than the expected value of $0.71 \mu_{b}$ for free $\mathrm{Sm}^{3+}$ ion. In many $\mathrm{Sm}$ compounds the saturation magnetic moment of $\mathrm{Sm}^{3+}$ is found to be significantly lower than the expected $0.71 \mu_{b}$ [58]. A close match between the expected and the observed moments, as obtained through magnetic-structure refinement, confirms the correctness of the magnetic structure obtained from Rietveld refinement of the low temperature TOF NPD data. 


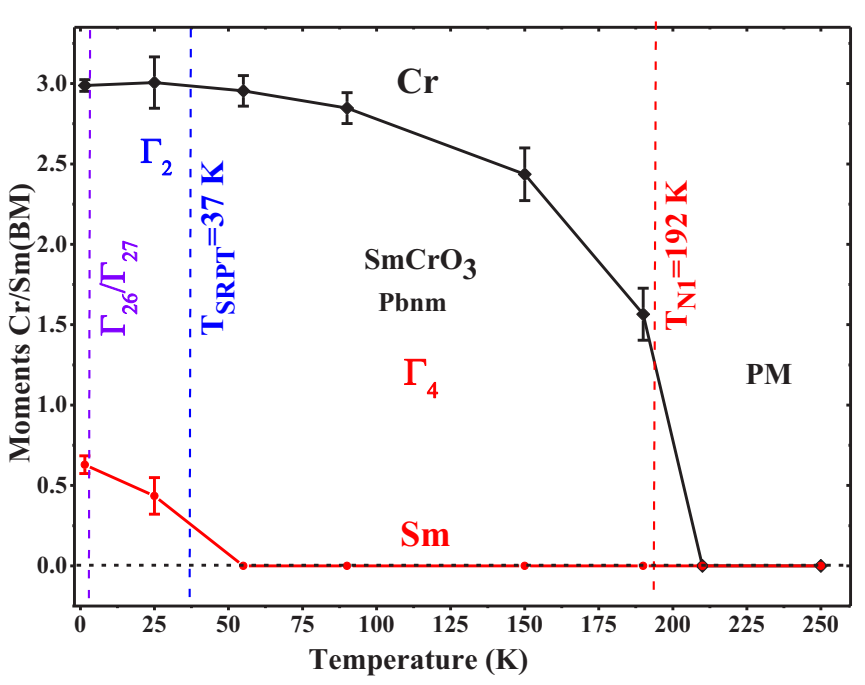

FIG. 9. Temperature dependence of the ordered moments of $\mathrm{Cr}^{3+}$ and $\mathrm{Sm}^{3+}$ as obtained from the constrained magnetic refinement.

Using the components of the $\mathrm{Cr}^{3+}$ moment as obtained through constrained refinement, canting angles (angle w.r.t. to the $a, b$, and $c$ axes) of the moment have been calculated. Figure 10 shows the temperature variation of the canting angles of $\mathrm{Cr}^{3+}$. It can be seen that, unlike in Ref. [26], the $\mathrm{Cr}^{3+}$ moments remain confined in the $a-b$ plane with major components aligned along the $\mathbf{a}$ axis in the $\Gamma_{4}$ phase, as proposed by Gorodetsky et al. [25]. It does not reveal any appreciable temperature dependence.

Looking at the $M-T$ variation in Fig. 3(a) it appears that the magnetocrystalline anisotropies of the $\Gamma_{2}$ and $\Gamma_{4}$ phases of $\mathrm{SmCrO}_{3}$ are way apart. Reversal of sign of second-order magnetic anisotropy across the SRPT has been known previously for rare-earth orthoferrites [8]. The magnetic-anisotropy

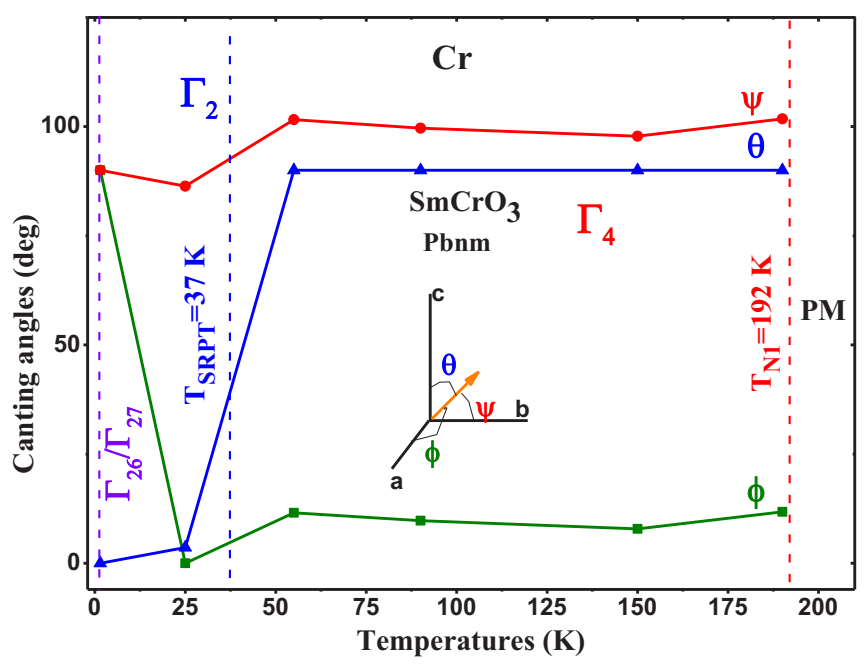

FIG. 10. Temperature variation of canting angles of the $\mathrm{Cr}$ and Sm moments w.r.t. the $a, b$, and $c$ axes in $\Gamma_{4}$ and $\Gamma_{2}$ phases. In the $\Gamma_{4}$ phase the $\mathrm{Cr}$ moments remain confined nearly in the $a$ - $b$ plane with the major component along the $a$ axis and flip along the $c$ axis below the SRPT. energy is given as Eq. (1).

$$
E=K_{0}+K_{1} \sin ^{2} \theta+K_{2} \sin ^{4} \theta .
$$

Here $K_{1}$ and $K_{2}$ are, respectively, the second- and fourthorder magnetic-anisotropy constants. Positive or negative $K$ ' $s$ would favor alignment of the moments along a particular axis (a.k.a. easy-axis type) or in a plane (a.k.a. easy-plane type), respectively. In the situation of $K^{\prime} s$ having opposite signs, easy-cone type anisotropy [59] is expected for $K_{2}=-\frac{1}{2} K_{1}$ to $K_{2}=\infty$ (with $K_{1}$ negative), which is practically realized as canted spins [60]. It has been theoretically shown [61] and investigated through microwave spectroscopy studies [62,63] that the SRPT arises due to fourth-order magnetic anisotropy, which is generally small but dominates the orientational behavior when the second-order magnetic anisotropy changes sign $[8,64,65]$.

Under the ZFC protocol of $M-T$ measurement the uncorrelated spins of $\mathrm{Cr}^{3+}$ moments in the paramagnetic state start aligning along their equilibrium orientations and order canted antiferromagnetically below the Néel temperature $T_{N 1}$. At $T_{N 1}$, however, the $\mathrm{Sm}^{3+}$ moments still remain uncorrelated. This CAFM state of $\mathrm{Cr}^{3+}$ moments, which has a resultant moment along the $z$ direction, generates an internal field. When the temperature is further lowered, the $\mathrm{Sm}^{3+}$ moments begin to respond to this internal field due to anisotropic superexchange interaction between them, and align antiparallel to it $[8,10$ $12,50,51,64,65]$. The interaction of $\mathrm{Sm}^{3+}$ moments with $\mathrm{Cr}^{3+}$ moments tries to rotate the $\mathrm{Cr}^{3+}$ moments along the $z$ direction. This process continues as continuous rotation of the $\mathrm{Cr}^{3+}$ moments leading to nearly $90^{\circ}$ flip at the SRPT [10]. From the refined spin-structure model given in Figs. 8(c) and 8(d) it is clear that the $\mathrm{Cr}^{3+}$ moments flip by $90^{\circ}$ below the SRPT. The $\mathrm{Sm}^{3+}$ moments also flip and maintain their nearly perpendicular orientation w.r.t. to the $\mathrm{Cr}^{3+}$ moments. Homer and Varma [9] have theoretically shown that under zero field, continuous rotation of moments may result due to temperature-dependent $K_{1}$ and $K_{2}$ magnetic-anisotropy constants leading to a secondorder phase transition. The continuous nature of rotation of $\mathrm{Cr}^{3+}$ moments is also experimentally realized through ZFC $M-T$ variation. As can be seen in Fig. SM 7 [36], the peak corresponding to the first derivative of ZFC $M-T$ is asymmetrically broadened around the SRPT. The derivative gradually rises from $\sim 53 \mathrm{~K}$, attains its peak at $34 \mathrm{~K}$ ( $19 \mathrm{~K}$ wide), and drops to its initial baseline at $26 \mathrm{~K}$, just within $8 \mathrm{~K}$. Due to continuous rotation of the $\mathrm{Cr}^{3+}$ moments its $\mathrm{FM}$ component keeps continuously increasing, and in response the antiparallel polarized $\mathrm{Sm}^{3+}$ moments also continuously increase, compensating the $\mathrm{Cr}^{3+} \mathrm{FM}$ component, so close to SRPT that the net magnetization starts decreasing. It is found in many rareearth chromates, that while cooled under optimum field, the overcompensation leads to negative magnetization. A much lower value of ZFC magnetization of the $\Gamma_{2}$ phase, as compared to $\Gamma_{4}$, could be due to high uniaxial magnetic anisotropy of the $\Gamma_{2}$ phase as compared to easy-plane anisotropy of the $\Gamma_{4}$ phase.

Under the FCC protocol, the presence of a magnetic field aids the moments to align along the field direction. Since in the $\Gamma_{4}$ phase the anisotropy is easy-plane type, the applied magnetic field overcomes the anisotropy, and orients the domain magnetization of the ferrimagnetic phase of $\mathrm{SmCrO}_{3}$ to 
TABLE III. Experimentally refined and DFT calculated moment vectors on various chromium sites for the $\Gamma_{1}$ and $\Gamma_{2}$ spin configurations of $\mathrm{SmCrO}_{3}$ at $1.5 \mathrm{~K}$.

\begin{tabular}{|c|c|c|c|c|}
\hline Site & Experimental & Calculated & Experimental & Calculated \\
\hline $\mathrm{Cr} 2$ & {$[-0.18,2.63,0.07]$} & {$[-0.05,2.84,0.05]$} & {$[0.14,-0.1,2.87]$} & {$[0.06,-0.04,2.84]$} \\
\hline $\mathrm{Cr} 3$ & {$[-0.18,2.63,-0.07]$} & {$[-0.06,2.84,-0.05]$} & {$[0.14,0.1,-2.87]$} & {$[0.04,0.04,-2.84]$} \\
\hline $\mathrm{Cr} 4$ & {$[0.18,2.63,-0.07]$} & {$[0.06,2.84,-0.04]$} & {$[0.14,0.1,2.87]$} & {$[0.06,0.05,2.84]$} \\
\hline
\end{tabular}

a certain extent along the field, resulting in higher magnetization in between $T_{N 1}$ and $T_{\mathrm{SRPT}}$ as compared to the ZFC case. The reversible drop in $M-T$ below $\sim 22 \mathrm{~K}$ indicates the commencement of the second-order phase transitions.

The sharp drop in $M-T$ below $\sim 10.6 \mathrm{~K}$, in contrast to the ZFC data, where the magnetization gradually increases with decrease in temperature, is attributed to the commencement of independent $A_{z}^{R}$ or $G_{z}^{R}$ type AFM ordering of $\mathrm{Sm}^{3+}$ with $T_{N 2} \sim 4 \mathrm{~K}$. The slight off-horizontal tilt of $\mathrm{Sm}^{3+}$ moments as shown in Figs. 8(a) and 8(b) stands for Sm-Sm independent AFM ordering. This observation corroborates very well with the specific-heat data on single-crystal $\mathrm{SmCrO}_{3}$ [25], showing commencement of Sm ordering at $\sim 10.6 \mathrm{~K}$, while it orders at $\sim 4 \mathrm{~K}$.

The $\sim 155 \mathrm{~K}$ wide irreversible features corresponding to the $M-T$ drop at $39 \mathrm{~K}$ appear to be a result of two different paths of the formation of the $\Gamma_{4}$ phase. During FCC the $\Gamma_{4}$ phase forms due to disorder-order transition under applied field, and hence formation of relatively oriented and larger ferrimagnetic domains leading to higher net magnetization is expected. On the other hand, during FCW the $\Gamma_{4}$ phase forms due to order-order transformation. As can be seen in Fig. 8(c) the exact horizontal orientation of $\mathrm{Sm}^{3+}$ moments in $\boldsymbol{C}_{\boldsymbol{y}}^{\boldsymbol{R}} \mathrm{AFM}$ configuration in the $\Gamma_{2}$ phase has two possible orientations to

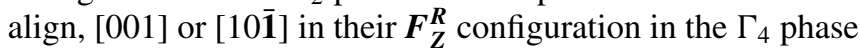
across the $\Gamma_{2}$ to $\Gamma_{4}$ phase transition during FCW; see Fig. 8(c). Hence the definition of preexisting domains will modify with $180^{\circ}$ domains of ferrimagnetic phase to minimize the magnetostatic energy cost. The increased number of $180^{\circ}$ domains will therefore lower the net magnetization. Since all domain structures ultimately vanish at the paramagnetic phase, the FCW $M-T$ gradually merges to FCC above $T_{N 1}=192 \mathrm{~K}$.

\section{Ground-state energy calculation for $\Gamma_{1}$ and $\Gamma_{2}$ spin configurations}

The experimental result of NPD showing transformation of $\Gamma_{4}$ spin configuration to $\Gamma_{2}$ below SRPT in $\mathrm{SmCrO}_{3}$ is in contradiction with the $\Gamma_{4}$ to $\Gamma_{1}\left(A_{x}, G_{y}, C_{Z} ; C_{z}^{R}\right)$ transformation as reported in Ref. [26]. This discrepancy has been independently probed through ground-state energy calculation for $\Gamma_{2}$ and $\Gamma_{1}$ spin configurations for $\mathrm{SmCrO}_{3}$. In order to understand the relative stabilities and predict correct spin configuration, the energies of low-temperature experimental crystal structures, with different spin configurations, were computed within the framework of density functional theory (DFT) as implemented in the VASP code [66-68] by employing the generalized gradient approximation (GGA) for exchange correlation and the rotationally invariant approach introduced by Perdew et al. [69,70] and Dudarev et al. [71] for explicit treatment of $\mathrm{Cr} 3 d$ electrons. Electron configurations of $5 s^{2}, 5 p^{6}, 5 d^{1}$, and $6 s^{2}$ for samarium; $3 p^{6}, 3 d^{5}$, and $4 s^{1}$ for chromium; and $6 s^{2}$ and $2 p^{6}$ for oxygen were treated as valence electrons. As the intraorbital electron correlations are known to be about 3-5 eV in transition-metal oxides [52,72], $U=3.7 \mathrm{eV}$ and $J=0.6 \mathrm{eV}$ were used in the calculation. Spin-orbit interactions were considered and all the symmetries were turned off in all the calculations. Plane waves up to $600 \mathrm{eV}$ were used and the total energies were converged to $10^{-6} \mathrm{eV} /$ u.c. during the electronic minimization. The experimental lattice parameters were used in all the calculations and ionic relaxation was carried out until a force tolerance of $0.005 \mathrm{eV} / \AA$ A was achieved. A $\Gamma$-centered $6 \times 6 \times 4 k$-point set was used to calculate the energies.

The unit cell of $\mathrm{SmCrO}_{3}$ contains four formula units with $\mathrm{Cr}^{3+}$ ions occupying edge- and face-center positions in an octahedral environment of $\mathrm{O}^{2-}$ ions. A G-type AFM state corresponding to $\Gamma_{1}$ and $\Gamma_{2}$ spin configurations was enforced by aligning moment vectors on the $\mathrm{Cr}^{3+}$ sites along the experimental directions as given in Table III. After ionic relaxation and subsequent electronic minimization the relaxed crystal structures were found identical to each other, except that the spin configurations were different. $\mathrm{The} \mathrm{Cr}^{3+}$ moments became relaxed and almost collinear to the $\mathbf{b}$ axis for $\Gamma_{1}$ and to the $\mathbf{c}$ axis for $\Gamma_{2}$ configurations; see Figs. 11(a) and 11(b). In both the cases, the calculated bond lengths are within $0.5 \%$ of the experimentally observed value of $1.98 \AA$. A comparison of the Rietveld refined $1.5 \mathrm{~K}$ crystal structure of $\mathrm{SmCrO}_{3}$ and the relaxed crystal structure obtained after electronic minimization is shown in the Supplemental Material [36]. The directions of

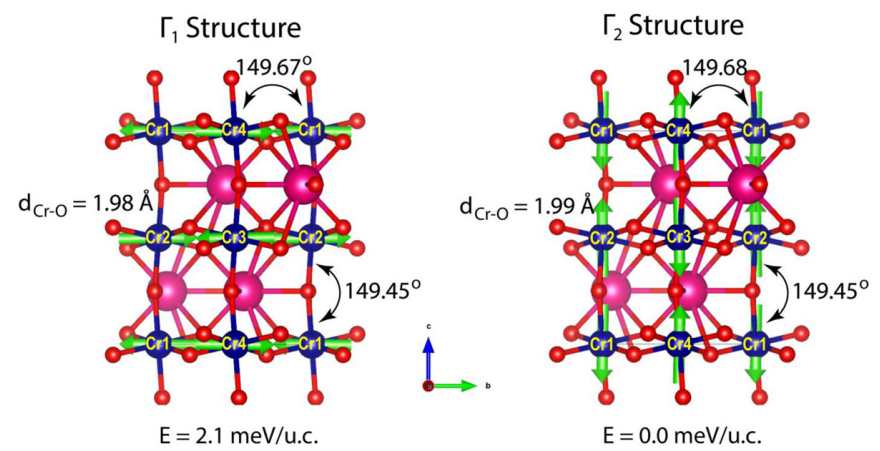

FIG. 11. DFT relaxed unit cells of $1.5 \mathrm{~K}$ structures with (a) $\Gamma_{1}$ and (b) $\Gamma_{2}$ spin configurations. The energy of the $\Gamma_{1}$ spin configuration is presented taking that of the $\Gamma_{2}$ as the reference. 
the moments as obtained after the neutron structure refinement and those obtained after DFT electronic minimization are both presented in Table III. The calculated moments show a small canting of spins, which is fully consistent with the $\mathrm{Cr}^{3+}$ moments obtained from Rietveld refinements. Among the two $1.5 \mathrm{~K}$ structures, the energy of the $\Gamma_{2}$ spin configuration is about $2.1 \mathrm{meV} /$ u.c. $(\sim 24 \mathrm{~K})$ lower than that of the $\Gamma_{1}$. Hence we conclude that the $\Gamma_{2}$ spin configuration as also obtained by the Rietveld refinement is the correct spin configuration of $\mathrm{SmCrO}_{3}$ below the SRPT.

\section{CONCLUSION}

Based on the combined dc-magnetization and highresolution TOF NPD studies we show that $\mathrm{SmCrO}_{3}$ undergoes a canted antiferromagnetic phase transition at $T_{N 1}=192 \mathrm{~K}$ with the $\mathrm{Cr}^{3+}$ and $\mathrm{Sm}^{3+}$ ions getting ordered to a spin configuration $\mathrm{Pb}^{\prime} n^{\prime} m: \Gamma_{4}\left(\boldsymbol{G}_{\boldsymbol{x}}, A_{y}, F_{Z} ; F_{Z}^{R}\right)$. The weak ferromagnetism across $T_{N 1}$ is due to uncompensated ferromagnetic components $F_{z}$ and $F_{z}^{R}$, respectively, of ordered $\mathrm{Cr}^{3+}$ and $\mathrm{Sm}^{3+}$ moments. At still lower temperatures the $\mathrm{Cr}^{3+}$ moments undergo continuous rotation, which causes SRPT at $37 \mathrm{~K}$ and the spin configuration changes from $P b^{\prime} n^{\prime} m: \Gamma_{4}\left(G_{x}, A_{y}, F_{Z} ; F_{Z}^{R}\right)$ to $P b n^{\prime} m^{\prime}: \Gamma_{2}\left(F_{x}, C_{y}, \boldsymbol{G}_{\boldsymbol{Z}} ; F_{x}^{R}, \boldsymbol{C}_{\boldsymbol{y}}^{\boldsymbol{R}}\right)$. At temperatures below $10 \mathrm{~K}$ the $\mathrm{Sm}^{3+}$ moments start to AFM align due to direct
Sm-Sm interaction against the internal field of $\mathrm{Cr}^{3+}$ and the $P b n^{\prime} m^{\prime}: \Gamma_{2}$ phase undergoes a second-order phase transition below $T_{N 2}<4 \mathrm{~K}$, resulting in either $\Gamma_{26}$ or $\Gamma_{27}$ spin configurations with polar order $P 2_{1}^{\prime} 2_{1}^{\prime} 2_{1}:\left(C_{x}, G_{\boldsymbol{y}}, F_{z} ; \boldsymbol{C}_{\boldsymbol{x}}^{\boldsymbol{R}}, A_{y}^{R}, F_{z}^{R}\right)$ or $P n^{\prime} a 2_{1}^{\prime}:\left(C_{x}, F_{y}, \boldsymbol{G}_{z} ; \boldsymbol{C}_{\boldsymbol{x}}^{\boldsymbol{R}}, \boldsymbol{F}_{\boldsymbol{y}}^{\boldsymbol{R}}, G_{z}^{R}\right), \quad$ respectively. The spin-dependent DFT calculation taking into account the spin-orbit interaction confirms that in $\mathrm{SmCrO}_{3}$ the $\Gamma_{2}\left(F_{x}, C_{y}, \boldsymbol{G}_{Z} ; F_{x}^{R}, C_{y}^{R}\right)$ spin configuration is lower in ground-state energy by $\sim 2.1 \mathrm{meV} /$ u.c. $(\sim 24 \mathrm{~K})$ as compared to that of the $\Gamma_{1}\left(A_{x}, G_{y}, C_{Z} ; C_{z}^{R}\right)$ spin configuration.

\section{ACKNOWLEDGMENTS}

The authors gratefully acknowledge Alok Banerjee for helpful discussions and magnetization data, and A. K. Srivastava and Pragya Tiwari RRCAT, Indore for providing EDS data. The authors are thankful to the Science and Technology Facilities Council (STFC), UK for providing beam time allocation to perform TOF-NPD measurements at the WISH, ISIS neutron and muon source. Travel support for P.Y. and T.S., respectively, from Newton India fund and the India-RAL project is sincerely acknowledged. S.S. gratefully acknowledges the Department of Science and Technology (DST), India for providing a postdoctoral fellowship through the Indo-UK Nano Mission project. V.P. would like to acknowledge the Czech Science Foundation (Project No. 18-10504S) for developing the JANA software.
[1] T. Kimura, T. Goto, H. Shintani, K. Ishizaka, T. Arima, and Y. Tokura, Nature 426, 55 (2003).

[2] T. Kimura, G. Lawes, Y. Tokura, T.Goto, and A. P. Ramirez, Phys. Rev. B 71, 224425 (2005).

[3] Y. Tokunaga, S. Iguchi, T. Arima, and Y. Tokura, Phys. Rev. Lett. 101, 097205 (2008).

[4] T. Yamaguchi and K. Tsushima, Phys. Rev. B 8, 5187 (1973).

[5] B. Rajeswaran, D. I. Khomskii, A. K. Zvezdin, C. N. R. Rao, and A. Sundaresan, Phys. Rev. B 86, 214409 (2012).

[6] S. Mahana, B. Rakshit, R. Basu, S. Dhara, B. Joseph, U. Manju, S. D. Mahanti, and D. Topwal, Phys. Rev. B 96, 104106 (2017).

[7] S. Mahana, U. Manju, P. Nandi, E. Welter, K. R. Priolkar, and D. Topwal, Phys. Rev. B 97, 224107 (2018).

[8] R. L. White, J. Appl. Phys. 40, 1061 (1969).

[9] H. Homer and C. M. Varma, Phys. Rev. Lett. 20, 845 (1968).

[10] T. Yamaguchi, J. Phys. Chem. Solids 35, 479 (1974).

[11] K. Yoshii, J. Solid State Chem. 159, 204 (2001).

[12] K. Yoshii, Mater. Res. Bull. 47, 3243 (2012).

[13] H. J. Zhao, J. Íñiguez, X. M. Chen, and L. Bellaiche, Phys. Rev. B 93, 014417 (2016).

[14] A. Durán, R. Escamilla, R. Escudero, F. Morales, and E. Verdín, Phys. Rev. Mater. 2, 014409 (2018).

[15] A. Kumar and S. M. Yusuf, Phys. Rep. 556, 1 (2015).

[16] S. Biswas and S. Pal, Rev. Adv. Mater. Sci. 53, 206 (2018).

[17] L. H. Yin, J. Yang, X. C. Kan, W. H. Song, J. M. Dai, and Y. P. Sun, J. Appl. Phys. 117, 133901 (2015).

[18] N. Kumar and A. Sundaresan, Solid State Commun. 150, 1162 (2010).

[19] Y. Cao, S. Cao, W. Ren, Z. Feng, S. Yuan, B. Kang, B. Lu, and J. Zhang, Appl. Phys. Lett. 104, 232405 (2014).
[20] Y. K. Jeong, J.-H. Lee, S.-J. Ahn, and H. M. Jang, Solid State Commun. 152, 1112 (2012).

[21] A. Kimel, B. Ivanov, R. Pisarev, P. Usachev, A. Kirilyuk, and T. Rasing, Nat. Phys. 5, 727 (2009).

[22] M. Shao, S. Cao, S. Yuan, J. Shang, B. Kang, B. Lu, and J. Zhang, Appl. Phys. Lett. 100, 222404 (2012).

[23] A. Ghosh, K. Dey, M. Chakraborty, S. Majumdar, and S. Giri, Europhys. Lett. 107, 47012 (2014).

[24] G. N. P. Oliveira, R. C. Teixeira, R. P. Moreira, J. G. Correia, J. P. Araújo, and A. M. L. Lopes, Sci. Rep. 10, 4686 (2020).

[25] G. Gorodetsky, R. Hornreich, S. Shaft, B. Sharon, A. Shaulov, and B. Wanklyn, Phys. Rev. B 16, 515 (1977).

[26] M. Tripathi, R. J. Choudhary, and D. M. Phase, Phys. Rev. B 96, 174421 (2017).

[27] E. F. Bertaut, G. Bassi, G. Buisson, P. Burlet, J. Chappert, J. Mareschal, A. Delapalme, G. Roult, R. Aleonard, R. Pauthenet et al., J. Appl. Phys. 37, 1038 (1966).

[28] E. F. Bertaut and J. Mareschal, Solid State Commun. 5, 93 (1967).

[29] E. F. Bertaut, Acta Crystallogr., Sect. A 24, 217 (1968).

[30] N. Shamir, H. Shaked, and S. Shtrikman, Phys. Rev. B 24, 6642 (1981).

[31] R. D. Pierce, R. Wolfe, and L. G. Van Uitert, J. Appl. Phys. 40, 1241 (1969).

[32] R. D. Johnson, N. Terada, and P. G. Radaelli, Phys. Rev. Lett. 108, 219701 (2012).

[33] J.-H. Lee, Y. K. Jeong, J. H. Park, M.-A. Oak, H. M. Jang, J. Y. Son, and J. F. Scott, Phys. Rev. Lett. 107, 117201 (2011).

[34] J.-H. Lee, Y. K. Jeong, J. H. Park, M.-A. Oak, H. M. Jang, J. Y. Son, and J. F. Scott, Phys. Rev. Lett. 108, 219702 (2012). 
[35] C.-Y. Kuo, Y. Drees, M. T. Fernández-Díaz, L. Zhao, L. Vasylechko, D. Sheptyakov, A. M. T. Bell, T. W. Pi, H.-J. Lin, M.-K. Wu, E. Pellegrin, S. M. Valvidares, Z. W. Li, P. Adler, A. Todorova, R. Küchler, A. Steppke, L. H. Tjeng, Z. Hu, and A. C. Komarek, Phys. Rev. Lett. 113, 217203 (2014).

[36] See Supplemental Material at http://link.aps.org/supplemental/ 10.1103/PhysRevB.103.144418, for the arguments in support of this work in the form of extra figures and tables, and some extra characterization data.

[37] L. C. Chapon, P. Manuel, P. G. Radaelli, C. Benson, L. Perrott, S. Ansell, N. J. Rhodes, D. Raspino, D. Duxbury, E. Spill, and J. Norris, Neutron News 22, 22 (2011).

[38] V. Petricek, M. Dusek, and L. Palatinus, Z. Kristallogr. - Cryst. Mater. 229, 345 (2014).

[39] Z. Xiang, W. Li, and Y. Cui, RSC Adv. 8, 8842 (2018).

[40] Z. Xiang, J. Xu, Y. Huang, S. Ge, and Y. Cui, Prog. Nat. Sci.: Mater. Int. 28, 609 (2018).

[41] M. Tripathi, R. J. Choudhary, and D. M. Phase, RSC Adv. 6, 90255 (2016).

[42] M. C. Biesinger, C. Brown, J. R. Mycroft, R. D. Davidson, and N. S. McIntyre, Surf. Interface Anal. 36, 1550 (2004).

[43] I. Ikemoto, K. Ishii, I. Kinoshita, H. Kuroda, M. A. Alario Franco, and J. M. Thomas, J. Solid State Chem. 17, 425 (1976).

[44] G. Dufour, R. C. Karnatak, J. M. Mariot, and C. Bonnelle, Chem. Phys. Lett. 42, 433 (1976).

[45] J. F. Moulder, W. F. Stickle, P. E. Sobol, and K. D. Bomben, Handbook of X-Ray Photoelectron Spectroscopy (Perkin-Elmer, Eden Prairie, MN, 1992), pp. 148-149.

[46] T. Morishita and K. Tsushima, Phys. Rev. B 24, 341 (1981).

[47] S. Lei, L. Liu, C. Wang, C. Wang, D. Guo, S. Zeng, B. Cheng, Y. Xiao, and L. Zhou, J. Mater. Chem. A 1, 11982 (2013).

[48] I. Dzyaloshinsky, J. Phys. Chem. Solids 4, 241 (1958).

[49] T. Moriya, Phys. Rev. 120, 91 (1960).

[50] L. Bellaiche, Z. Gui, and I. A. Kornev, J. Phys.: Condens. Matter 24, 312201 (2012).
[51] D. I. Khomskii, J. Magn. Magn. Mater. 306, 1 (2006).

[52] X. Qian, L. Chen, S. Cao, and J. Zhang, Solid State Commun. 195, 21 (2014).

[53] N. Shamir, H. Shaked, and S. Shtrikman, Physica B+C (Amsterdam) 90, 211 (1977).

[54] E. Wollan and W. Koehler, Phys. Rev. 100, 545 (1955).

[55] E. Bousquet and A. Cano, J. Phys.: Condens. Matter 28, 123001 (2016).

[56] B. J. Campbell, H. T. Stokes, D. E. Tanner, and D. M. Hatch, J. Appl. Crystallogr. 39, 607 (2006).

[57] G. Gorodetsky, R. M. Hornreich, I. Yaeger, H. Pinto, G. Shachar, and H. Shaked, Phys. Rev. B 8, 3398 (1973).

[58] S. K. Malik and R. Vijayaraghavan, Pramana 3, 122 (1974).

[59] B. D. Cullity and C. D. Graham, Introduction to Magnetic Materials, 2nd ed. (John Wiley, New York, 2009), pp. 197-210.

[60] A. A. Timopheev, R. Sousa, M. Chshiev, H. T. Nguyen, and B. Dieny, Sci. Rep. 6, 26877 (2016).

[61] G. F. Herrmann, Phys. Chem. Solids 24, 597 (1963).

[62] F. B. Hagedorn and E. M. Gyorgy, Phys. Rev. 174, 540 (1968).

[63] E. M. Gyorgy, J. P. Remeika, and F. B. Hagedorn, J. Appl. Phys. 39, 1369 (1968).

[64] J.-S. Zhou, J. A. Alonso, V. Pomjakushin, J. B. Goodenough, Y. Ren, J.-Q. Yan, and J.-G. Cheng, Phys. Rev. B 81, 214115 (2010).

[65] M. Taheri, F. S. Razavi, Z. Yamani, R. Flacau, P. G. Reuvekamp, A. Schulz, and R. K. Kremer, Phys. Rev. B 93, 104414 (2016).

[66] G. Kresse and J. Furthmuller, Comput. Mater. Sci. 6, 15 (1996).

[67] P. E. Blöchl, Phys. Rev. B 50, 17953 (1994).

[68] G. Kresse and D. Joubert, Phys. Rev. B 59, 1758 (1999).

[69] J. P. Perdew, K. Burke, and M. Ernzerhoff, Phys. Rev. Lett. 77, 3865 (1996).

[70] J. P. Perdew, K. Burke, and M. Ernzerhoff, Phys. Rev. Lett. 78, 1396(E) (1997).

[71] S. L. Dudarev, G. A. Botton, S. Y. Savrasov, C. J. Humphreys, and A. P. Sutton, Phys. Rev. B 57, 1505 (1998).

[72] S. R. Barman and D. D. Sarma, Phys. Rev. B 49, 13979 (1994). 